Aus der Klinik für Allgemeine Chirurgie, Viszeral-, Gefäß- und Kinderchirurgie

Universitätsklinikum des Saarlandes, Homburg/Saar

Direktor Prof. Dr. Dr. med. Martin K. Schilling

\title{
Arbeitszeitkonformes Visitenmodell und
}

\section{Patientenzufriedenheit}

\author{
Dissertation \\ zur Erlangung des Grades eines Doktors der Medizin \\ der Medizinischen Fakultät der Universität des Saarlandes
}

2010

vorgelegt von

Sandra Veigel

geb. am 25.09.1978 in Tübingen 


\section{Inhaltsverzeichnis}

1. Zusammenfassung

2. Summary

3. Einleitung

3.1. Definition

3.2. Historische Angaben

3.3. Bedeutung und Funktion der Visite 8

3.4. Visitenarten 9

3.4.1. Kurvenvisite 9

3.4.2. Stationsarztvisite 9

3.4.3. Oberarztvisite 10

3.4.4. Chefarztvisite 11

3.5. Rituale und ihre Bedeutung zur Visite 11

3.6. Arbeitszeitgesetz und klinische Patientenpfade 12

4. Methodik 15

4.1. Studiendesign 15

4.2. Patientenrekrutierung $\quad 15$

$\begin{array}{ll}\text { 4.3. Ausschlußkriterien } & 16\end{array}$

4.4. Präoperative Patientenbefragung 16

$\begin{array}{ll}\text { 4.5. Visitenbeobachtung } & 18\end{array}$

4.6. Patienteninterview 21

4.7. Auswertung und Statistik 25

4.8. Ausscheidungen und Studienabbrüche 25 
5. Ergebnisse 26

5.1. Demographische und klinische Daten 26

5.2. Präoperative Patientenerwartung $\quad 27$

5.3. Visitenbeobachtung und Patientenzufriedenheit 30

5.4. Patientenresonanzen 48

5.5. Geschlechtsvergleich 48

6. Diskussion $\quad \mathbf{5 0}$

6.1. Allgemeines 50

6.2. Umstrukturierung Visitenführung 52

6.3. Clinical pathways 52

6.4. Umsetzung des Arbeitsschutzgesetzes und Patientenzufriedenheit 54

6.5. Fazit 55

$\begin{array}{ll}\text { 7. Literatur } & 57\end{array}$

8. Abkürzungsverzeichnis 62

9. Abbildungsverzeichnis 63

10. Tabellenverzeichnis 64

11. Publikation 65

12. Lebenslauf 66

$\begin{array}{ll}\text { 13. Danksagung } & 68\end{array}$ 


\section{Zusammenfassung}

\section{Hintergrund:}

Die Umsetzung des Arbeitszeitgesetzes vom ersten Januar 2006 forderte von vielen Krankenhäusern weitere Rationalisierungen im stationären Arbeitsablauf. Trotz erfolgreicher Einführung von Patientenpfaden (clinical pathways (CPs)) waren weitere rationalisierende Maßnahmen, mitunter eine drastische Kürzung der stationären Morgenvisite erforderlich. Hieraus ergab sich die Schwierigkeit einen effektiven Arbeitsablauf unter Berücksichtigung von Patientenbedürfnissen zu gewährleisten.

\section{Methodik:}

Seit Januar 2009 wurde die Visitendauer von 45 auf 30 min gekürzt. Stationsoberärzte wurden gleichzeitig in den täglichen Visitenablauf eingebunden. Die prospektiv angelegte Studie wurde vom März bis Juni 2009 durchgeführt. Hierbei wurde ein Stationsbereich mit einer maximalen Kapazität von 23 Betten mit ausschließlich viszeral- und gefäßchirurgischen Patienten ausgesucht. In diesem Zeitraum konnten 86 Patienten ausgewertet werden. In der Studie wurde ein Beobachtungsfenster vom 1. bis zum 5. postoperativen Tag (POT) und am Entlassungstag (ET) festgelegt. Alle Patienten wurden anhand der Länge ihres stationären Aufenthaltes in 2 Gruppen eingeteilt. Gruppe 1 umfasst das Patientenkollektiv mit einer Verweildauer von > 5 (46 Probanden) und Gruppe 2 von $>1$ und $\leq 5$ (40 Probanden) stationären Krankenhaustagen.

\section{Ergebnisse:}

Es wurden 86 Patienten darunter 40 Frauen mit einem Durchschnittsalter von 56,7 Jahren in der Studie aufgenommen. Die Patienten hatten eine durchschnittliche stationäre Verweildauer von 7,2 Tagen. 69,8 \% der in dieser Untersuchung erfassten Patienten wurden als CPs erfasst. Die präoperative Patientenerwartung war mit 5,3 min signifikant höher als die tatsächlich ermittelten Werte. Eine ärztliche Begrüßung zur Visite erfolgte jeweils in beiden Gruppen in mehr als $95 \%$ zu Beginn des Visitengespräches. Allerdings gab es große Schwankungen hinsichtlich der ärztlichen Verabschiedung vom Patienten (Abweichung $32 \%$ - 86,7 \%). Hierbei zeigte sich ein signifikanter Unterschied in unserer Gruppe 1 am 2. POT versus 4. POT und versus ET.

Am 3. POT wurde eine körperliche Untersuchung innerhalb der Gruppe 1 mit $77,3 \%$ am häufigsten durchgeführt ( $p<0,05$ versus 5. POT), während in Gruppe 2 
am ET bei 63,0 \% der höchste Wert ermittelt werden konnte. Eine genaue Erklärung über den operativen Verlauf wurde signifikant häufiger am 1. POT in 55,6 \% der Fälle in Gruppe 1 durchgeführt ( $p<0,05$ versus 2. - 5. POT). In 56,5\% der Visitenbeobachtungen wurden am 1. POT in Gruppe 2 den Patienten Informationen zum intraoperativen Verlauf vermittelt. Durchschnittlich dauerten die Visiten innerhalb der Gruppe 1 am 3. POT 1,5 min und am ET 1,1 min. Die präoperative Patientenerwartung (PE) war allerdings mit 5,3 min signifikant höher ( $p<0,05$ versus 1. - 5. POT und ET). In Gruppe 2 belief sich die Visitenlänge auf 0,8 min am 1. POT und auf 0,9 min am ET bei von Patientenseite erwarteten $4,4 \mathrm{~min}$.

Das reine Arzt-Patientengespräch zur Visite war in Gruppe 1 am 1. POT mit 0,33 min signifikant niedriger als am ET mit 0,93 min. In Gruppe 2 gab es am 1. POT mit 0,49 min und am ET mit 0,68 min keinen signifikanten Unterschied. Die meisten ärztlichen Fragen an die Patienten zur Visite wurden am 3. POT (durchschnittlich 3,4 Fragen) in der Gruppe 1 ohne signifikanten Unterschied gestellt. An den anderen Tagen im Beobachtungsfenster erhoben die Ärzte am 1. und 2. POT durchschnittlich 2 Fragen und am 4. bis 5. POT und am ET mit 2,4 bis 2,5 annähernd gleiche Anzahl an Fragen an die Patienten. Verglichen mit Gruppe 1 wurde in Gruppe 2 an beiden Beobachtungstagen eine weniger intensive ärztliche Befragung mit 1,5 versus 1,9 Fragen durchgeführt. Bei der Auswertung des Frageninhaltes zeigte sich, dass am 1. POT in $77,8 \%$, respektive $73,9 \%$ der Fälle in Gruppe 1 und 2 nach postoperativen Schmerzen gefragt wurde. Der Informationsbedarf der Patienten am ET in Gruppe 1 war signifikant im Vergleich zu den Tagen 2. bis 5. POT mit durchschnittlich 1,5 Fragen pro Visite erhöht.

In beiden Gruppen konnte eine Patientenzufriedenheit stets von mehr als 80 $\%$ erreicht werden. An positiver Resonanz nannten die Probanden am Häufigsten die gute Gesprächsführung und Gesprächsatmosphäre der ärztlichen Mitarbeiter zur Visite.

\section{Diskussion:}

Die erhobenen Daten aus unserer prospektiven Studie zur Erfassung der Patientenzufriedenheit nach Umstrukturierung der Visiten konnten zeigen, dass trotz deutlicher Kürzungen der Visitenzeiten dennoch eine hohe Patientenzufriedenheit auf einer chirurgischen Station durch Visitenbesetzung mit Entscheidungsträgern und der routinemäßigen Eingliederung der Patienten in $\mathrm{CPs}$, zu gewährleisten ist. 


\section{Summary}

\section{Background:}

The implementation of the Working Hours Act on January $1^{\text {st }}, 2006$ required further rationalization on the stationary workflow process from many units. Although the clinical pathways (CPs) have been implemented successfully further rationalization steps became necessary, thereunder drastic reduction of the stationary morning ward rounds. As a consequence it was difficult to find the balance between the ensurance of an effective workflow processing and at the same time to meet the patients' interests during the ward round.

\section{Methods:}

By January 2009 the time frame for daily ward rounds has been reduced from 45 to $30 \mathrm{~min}$. The senior physicians equally have been integrated into the daily ward round processing. This prospective investigation was carried out from March until June 2009. The selection was made on a medical care unit with capacity of maximum 23 beds with exclusively visceral and vascular surgical patients. During this time frame datas of 86 patients could be evaluated. The investigation defined two observation periods: the first observation period covered postoperative day 1 until 5 ; the second observation period was related to the day of discharge. Based on the period of hospitalization, 2 different groups of patients have been set up. Group 1 consisting of 46 probands covered the stay of $>5$ days and group 2 with 40 probands the stay of $>1$ and $\leq 5$ days.

\section{Results:}

86 patients ( 46 male / 40 female) with an average age of 56.7 years have been admitted to this investigation. Their average in-station period lasted 7.2 days. $69.8 \%$ out of these patients were recorded as CPs. Preoperatively, the patients expected the ward round to last 5.3 min which was significantly higher than known from experience. Words of greetings by the physician towards the patient prior to the medical interview took place in more than $95 \%$ in both groups. However, great fluctuation of $32 \%$ up to $86.7 \%$ with regard to the formal discharge by the physician had to be noticed. A significant difference in group 1 between the $2^{\text {nd }}$ postoperative day versus $4^{\text {th }}$ postoperative day and vs. day of discharge was observed.

On postoperative day 3 , physical examination was done most frequently in group $1(77.3 \%, p<0.05$ versus postoperative day 5$)$ whereas patients of group 2 were most frequently physically examined on the day of discharge $(63.0 \%)$. Patients 
of group 1 were informed in detail about the course of the operation significantly more frequently on the $1^{\text {st }}$ postoperative day $\left(55.6 \%, p<0.05\right.$ versus $2^{\text {nd }}-5^{\text {th }}$ postoperative day). The evaluated rate with regard to the intraoperative information given to group 2 on postoperative day 1 amounted to $56.5 \%$. On average, the time spent on patients of group 1 during the ward round on postoperative day 3 lasted at longest $1.5 \mathrm{~min}$ and on the day of discharge $1.1 \mathrm{~min}$. Preoperatively, however, the patients expected a significantly longer lasting ward round of $5.3 \mathrm{~min}(\mathrm{p}<0.05$ versus $1^{\text {st }}-5^{\text {th }}$ postoperative day and day of discharge). The time for ward rounds spent on patients of group 2 came up to $0.8 \mathrm{~min}$ on the $1^{\text {st }}$ postoperative day and 0.9 min on the day of discharge. The expectation, however, was $4.4 \mathrm{~min}$.

The real patient-physician interview during the ward round in group 1 on postoperative day 1 lasting for a period of $0.33 \mathrm{~min}$ was significantly shorter compared to the day of discharge with $0.93 \mathrm{~min}$. With the evaluated result of 0.49 minutes on postoperative day 1 and $0.68 \mathrm{~min}$ on the day of discharge, no significant difference was recognized in group 2. Most of the questions addressed to the patients of group 1 during the ward round were asked at postoperative day 3 (averagely 3.4 questions) without statistical significance. $77.8 \%$ of the patients of group 1 and respectively $73.9 \%$ of group 2 demonstrated postoperative pain on postoperative day 1 . The information needs of the patients out of group 1 was significantly higher by 1.5 questions on average on the day of discharge compared with postoperative day 2 until 5 .

More than $80 \%$ of patients in both groups mentioned satisfaction.

\section{Discussion:}

Despite the reduced time spent on ward rounds, a high level of overall patient satisfaction can be obtained due to the regular attendance of a senior staff surgeon. Process management is furthermore endorsed by the routine application of clinical pathways in patient management. 


\section{Einleitung}

\subsection{Definition}

Der Begriff Visite leitet sich vom Lateinischen „visitare“ ab, das „besuchen“ und „hinzutreten“ bedeutet [12].

\subsection{Historische Angaben zur Visite}

Maaß hatte bereits verdeutlicht, dass die Visite schon immer teilweise konkurrierende und häufig unterschiedlich gewichtete Funktionen in sich vereinigt. Im frühen Altertum gab es weder bei den Griechen noch bei den Römern Krankenhäuser und somit auch keine Patientenvisiten [14]. Seit dem 4. Jahrhundert vor Christi wurden Kranke in Arzthäusern behandelt, von römischen Gutsbesitzern wurden Krankensäle zur Pflege von Sklaven eingerichtet und Heerlazarette dienten der Versorgung von Soldaten. Es existieren keine Überlieferungen zur Visite aus dieser Zeit. Im Jahr 1136 entstand in Konstantinopel ein Musterkrankenhaus, mit von Kaiser Johannes II. genau festgelegten Handlungsabläufen, u.a. auch mit Richtlinien zur Visite, die sowohl der Betreuung der Kranken als auch der Beaufsichtigung von Ärzten und Pflegepersonal diente [14]. In der arabischen Medizin, deren Zentrum zunächst in Damaskus lag, waren Krankenhausschulen schon um 900 vor Christi zur theoretischen und praktischen Ausbildung etabliert worden. In dieser Zeit stellte die Visite neben der Betreuung von Kranken bereits einen wichtigen Bestandteil des klinisch praktischen Unterrichts der damaligen Medizinstudenten dar [14]. Der Johanniterorden gründete im Jahre 1099 sein erstes Pflegehaus. Die Ordensvorschrift aus dem Jahre 1181 legte eine zweimalige Visite pro Tag mit schriftlicher Festhaltung der weiteren Prozedur durch einen Schreiber fest [14]. Im Abendland entstanden im frühen Mittelalter Pflegeeinrichtungen für Hilfsbedürftige aller Art. Krankenräume in Klöstern konnten im Laufe der Zeit von allen Menschen in Anspruch genommen werden. Vom 13. Jahrhundert an übernahmen viele Städte die Hospitäler von Klöstern oder gründeten selbst Krankenanstalten. Die damals angestellten Ärzte arbeiteten noch nicht regelmäßig in den Hospitälern, sondern sie wurden erst in besonderen Situationen zu einzelnen Patienten hinzu gerufen [14]. 
Regelmäßige Visiten fanden somit auch nicht statt. Auch in den folgenden Jahrhunderten gibt es keine Hinweise auf eine regelmäßige Visite von Erkrankten. Ende des 18. Jahrhunderts stellte die tägliche Visite im Wiener Dreifaltigkeitsspital eine große Errungenschaft dar. Die Zeit war von der Einstellung geprägt, dass es dem Staat nur gut gehen kann, wenn das Volk gesund ist, und aus diesem Grunde wurden mehr und mehr große Krankenhäuser gebaut. Die Visiten wurden täglich abgehalten und hatten eine überwiegend didaktische, diagnostische, aber auch therapeutische Funktion [14].

\subsection{Bedeutung und Funktion der Visite in der Literatur}

In mehreren Arbeiten wird die herausragende Bedeutung der Visite für die ArztPatientenbeziehung und die Funktion im Klinikalltag dargestellt.

Scherf fand in seiner Untersuchung heraus, dass die Visite ein wirksames und differenziertes diagnostisches Instrument darstellt [24]. Laut Rassek et al. ist die Visite die einzige, sich täglich zuverlässig wiederholende gemeinsame Gesprächsmöglichkeit [25]. Ein besonderes Interesse verdient die Visite deshalb, da sie sowohl für die Vermittlung von sachlichen Informationen als auch für emotionalen Beistand dient [22]. In der Arbeit von Westphal et al. wird zur Visite zwischen diagnostischen und therapeutischen Funktionen unterschieden, und letztgenannte in spezifisch und unspezifisch untergliedert. Als unspezifisch gelten Zuwendung und Interesse, die stabilisierend und stützend auf den Patienten wirken, und als spezifisch gelten dabei die Bewusstmachung und Bearbeitung von Konflikten [35]. Vogt beschreibt in ihrer Arbeit eine idealtypische Visite aus 7 patienten- und teambezogenen Funktionen. In dieser idealtypischen Visite werden Auftragsklärung und Gesamtplanung, Zwischenanamnese und Verlaufsbeobachtung, Entlassung und Prognose, Beziehungsgestaltung, Kontrolle, Supervision und Ausbildung sowie Informationsaustausch zwischen Teammitgliedern und Präsentation als Team vereint [36]. Bliesner und Köhle erarbeiteten neben den organisatorischen und diagnostischen Funktionen noch heraus, dass Kooperation, Compliance (Bereitschaft des Patienten zur Mitarbeit bei diagnostischen und therapeutischen Maßnahmen und ärztliche Anweisungen zu befolgen) und Coping (Bewältigungsverhalten des Patienten) durch die Visite gefördert werden können [3]. 
Aufgeteilt wird die Visite in den Ergebnissen von Fehlenberg et al. in arbeitsorganisatorische, institutionell bedingte und patientenbezogene Funktionen. Unter diagnostischen Tätigkeiten werden die Kontrolle des Behandlungseffekts und die Information des Patienten verstanden, womit dessen Compliance verbessert werden kann [6].

\subsection{Visitenarten}

Es gibt verschiedene Visitenarten, die unterschiedliche Anforderungen an den Arzt stellen, und gleichzeitig mit einer unterschiedlichen Erwartungshaltung seitens des Patienten vergesellschaftet sind [10].

\subsubsection{Kurvenvisite}

Die Kurvenvisite soll strikt von dem ärztlichen Gespräch mit dem Patienten getrennt werden [10]. In ihr sollen ausschließlich Untersuchungsergebnisse unter den ärztlichen Kollegen ggf. mit den Schwestern diskutiert werden, sowie medizinischfachliche, pharmakologische und / oder pflegerische Probleme thematisiert werden[10]. Räumlich kann die Kurvenvisite vor dem Patientenzimmer auf dem Flur oder im Arztzimmer abgehalten werden [10]. Einen Benefit durch die räumliche Trennung der Kurvenvisite von der eigentlichen Patientenvisite erzielt man durch die Möglichkeit in Fachtermini kommunizieren zu können und redundante Formulierungen vermeiden zu können [10].

\subsubsection{Stationsarztvisite}

In der Stationsarztvisite sind sowohl arbeitsorganisatorische und institutionelle Aufgaben als auch patientenbezogenen Aufgaben in sich zu vereinen [10,35]. Unter arbeitsorganisatorischen und institutionellen Aufgaben sind ärztliche Fachdiskussionen über Diagnostik, Diagnose und Therapie, Delegieren von Arbeitsaufgaben an das Fachpersonal und in begrenztem Umfang auch die 
Ausbildung von jungen ärztlichen Kollegen und Studenten zu verstehen [10]. Die patientenbezogenen Aufgaben umfassen die Untersuchung des Patienten zur Diagnosestellung, Kontrolle der Behandlungseffekte und Informationsweitergabe an den Patienten [10]. Es ist sinnvoll wenn der visiteführende Stationsarzt den überwiegenden Tei seiner Arbeitszeit auf der Station zubringt, um die Patienten und die jeweiligen Krankheitsverläufe so noch besser zu kennen [10]. Es wird empfohlen auf tägliche oder wöchentliche Wechsel in der Visitenführung der Stationsarztvisite zu verzichten [10]. Der visiteführende Stationsarzt soll in der Lage sein klare Anweisungen zu formulieren, ohne weitreichende Oberarzt- oder Chefarztentscheidungen, vorwegzunehmen [10].

\subsubsection{Oberarztvisite}

Eine Oberarztvisite kann auf ganz unterschiedliche Arten abgehalten werden, zwischen den beiden Extremformen gibt es zahlreiche Zwischenmöglichkeiten [10]. Entweder erfolgt eine Art Schnelldurchlauf von einem Patienten zum nächsten oder aber es wird im Anschluss an eine Kurvenvisite von oberärztlicher Seite noch eine Oberarztvisite inclusive Befragung des Patienten zum Befinden und Erläuterung des weiteren Procederes durchgeführt [10]. Die letztgenannte Möglichkeit der Durchführung einer oberärztlichen Visite wird als erfolgreicher eingestuft [10]. Bei dieser Art der Visitengestaltung ist vom Oberarzt indiziert, dass er seine fachliche Kompetenz offen darlegt, insbesondere Anordnungen erläutert und sich dem Patienten gegenüber gesprächsbereit zeigt [10]. Vom Stationsarzt setzt diese Visitenform eine intensive Vorbereitung voraus [10]. Der Stationsarzt muss hierbei Ratschläge und Kritik von oberärztlicher Seite annehmen, und nach gemeinsamer Diskussion von Problemen die anschließende Gesprächsführung mit dem Patienten, dem Oberarzt überlassen [10]. Von besonderer Bedeutung für den Patienten ist die Oberarztvisite bezüglich der Expertise des Oberarztes, ihm schwierige Sachverhalte und das weitere Procedere noch besser erläutern zu können [10]. Appelle an die Compliance des Patienten können vom Oberarzt schärfer formuliert werden, ohne dass die Vertrauensbasis zwischen Stationsarzt und Patient davon negativ beeinträchtigt wird [6]. 


\subsubsection{Chefarztvisite}

In noch größerem Maße als der Oberarzt stellt der Chefarzt der Klinik für Patienten die wichtigste Autorität in ärztlichem ebenso wie in zwischenmenschlichen Verhaltensweisen dar [10]. Bedingt ist dies durch die enorme Lebenserfahrung und das meist vorangeschrittene Lebensalter des Chefarztes im Vergleich zu Oberärzten und Stationsärzten [10]. Die Chefartzvisite kann analog zur Oberarztvisite zwischen den beiden Extremen eines Schnelldurchlaufs über die Station oder einer detailierten Kurven- und anschließenden Patientenvisite durchgeführt werden [10]. Hervorgehoben kann die Sonderstellung des Chefarztes in den normalen Stationsarztvisiten durch Hinweis auf die besondere Kompetenz des Chefarztes, sowie der Vorbehalt wichtige Entscheidungen ausschließlich vom Chefarzt zu treffen [10]. Die Gesprächs-Taktik soll im Interesse der Patienten vorher zwischen Stationsarzt, Oberarzt und Chefarzt abgesprochen werden, um eine synergistische Patientenberatung durchführen zu können [10]. Als vorteilhaft einer Chefarztvisite ist die Tatsache anzusehen, dass komplizierte Sachverhalte in der Visite zwischen den Entscheidungsträgern besprochen werden können, und so dem Patienten der Eindruck von Präsenz und gleichzeitig Geschlossenheit demonstriert werden kann [36]. Nachteilig an der Chefarztvisite kann sich auswirken, dass einzig die Meinung des Chefarztes als richtig angesehen wird, die Zahl der Personen zur Visite sich erhöht, und eher oberflächliche Visiten abgehalten werden, um längere Diskussionen zu vermeiden [10]. Aufgabe des Chefarztes ist es auch im Rahmen der Chefarztvisite seine große berufliche Erfahrung, seine fachlichen Kenntnisse und speziell auch sein Wissen zur menschenorientierten Führung bzw. Patientenbehandlung vorbildhaft an seine Mitarbeiter weiterzugeben [10].

\subsection{Rituale und ihre Bedeutung zur Visite}

In diesem Zusammenhang ist unter dem Begriff „Ritual“ eine expressive institutionalisierte Handlung oder Handlungssequenz zu verstehen [10].

Mit Ritualen soll die Asymmetrie im ärztlichen Gespräch überwunden werden. Dadurch soll dem Patienten sowohl verbal als auch nonverbal Bestätigung und Anerkennung entgegengebracht werden, und ihm gleichzeitig das Gefühl von 
Sicherheit und Geborgenheit vermittelt werden [10]. Der Visitenablauf soll mit Hilfe von Ritualen sowohl für den Arzt als auch für den Patienten klar strukturiert werden [10]. Durch den sich täglich wiederholenden gleichen Visitenablauf wird dieser für den Patienten berechenbarer und somit kann der Patient selbigem entspannter entgegensehen [21, 25].

Eröffnet soll die Visite mit einem Begrüßungsritual werden, das meist direkt in das Ritual der Mittelphase übergeht, in dem charakteristischerweise nach dem Befinden des Patienten gefragt wird [10]. Sollte es im Rahmen der Visite indiziert sein eine körperliche Untersuchung des Patienten durchzuführen, ist darauf zu achten, den Patienten vorher diesbezüglich zu informieren und zu fragen, im Sinne eines Rituals zum Eingriff in die Privatsphäre [10]. Durch bestätigende Rituale seitens des Arztes kann der Patient animiert werden weiter seine Beschwerden zu erläutern [10]. Empathierituale dienen dazu Mitgefühl und Verständnis für den Patienten auszudrücken, dies ist für eine vertrauensvolle symmetrische Arzt-PatientBeziehung von großer Bedeutung [10]. Unsicherheitsrituale sind grundsätzlich zu vermeiden [10]. Sie können sowohl auf verbaler als auch auf nonverbaler Ebene durch den Arzt geäußert werden, und den Patienten verunsichern [10]. In diesem Zusammenhang ist sicherlich indiziert dem Patienten die Grenzen ärztlichen Handelns und der medizinischen Möglichkeiten aufzuzeigen [10]. Durch die Abschiedssequenz leitet der Arzt das nahende Gesprächsende ein, darin soll dem Patienten die Gelegenheit gegeben werden Fragen zu äußern [10]. Letztlich beendet wird die Visite mit einem Abschiedsritual, das idealerweise analog zur Begrüßung mit einem Handschlag verbunden ist, um so das Visitenende auf zweifache Art für den Patienten zu markieren[10]. Sehr häufig äußert der Patient im Anschluss an das Abschiedsritual seinen Dank an den Arzt [10].

\subsection{Arbeitszeitgesetz und klinische Patientenpfade}

Die Visite stellt während des stationären Aufenthalts eine zentrale Rolle für den Patienten dar. Zwischen Patient und Arzt ist diese oft die einzige regelmäßige Gesprächsmöglichkeit während des stationären Aufenthalts [21]. Von daher wird der Visite eine hohe Erwartungshaltung entgegen gebracht. Bisherige Untersuchungen konnten oftmals zahlreiche Defizite im Arzt-Patient Dialog zeigen [8, 13]. Bliesner 
und Köhle nennen die traditionelle Visite schlichtweg einen „verhinderten Dialog“ [3]. Das Visitengespräch ist in der Regel thematisch ein fachliches Gespräch, in dem der letztlich „inkompetente Patient“ sich mit hochkompetenten Gesprächspartnern konfrontiert sieht [8]. Dabei gilt es einen Interessenskonflikt zwischen den Patientenbedürfnissen und einer zeitlich rationalisierten Visite seitens der Ärzte, insbesondere auf chirurgisch geführten Stationen, auszugleichen. Die bislang durchgeführten Recherchen ergaben, dass Patienten vor allem die Kürze der Visiten als negativ empfanden, worunter auch die Gesamtzufriedenheit abnahm [32]. Dieser Konflikt wird auf nationaler Ebene durch die zunehmende Anzahl behandelnder Patienten und der damit höheren Belastung der Ärzte und des Pflegepersonals bei unverändertem Personalschlüssel verschärft [23]. Nach Umsetzung des Arbeitszeitgesetzes (ArbZG) vom ersten Januar 2006 sind viele Abteilungen zu weiteren Rationalisierungen und Kürzungen im stationären Arbeitsablauf gezwungen $[31,32]$.

Eines der Elemente in unserer allgemeinchirurgischen Klinik zur strukturierten Ablauforganisation waren die Einführungen von Patientenpfaden (clinical pathways, CPs) für operativ interventionelle und konservative Behandlungsabläufe [26]. Schuld et al. konnten hierbei neben objektivierbaren ökonomischen Aspekten auch die subjektiv empfundene Zufriedenheit der behandelten Patienten als Grundlage einer strategischen Krankenhausführung belegen. Trotz erfolgreicher Einführung der CPs waren weitere rationalisierende Maßnahmen, mitunter eine drastische Kürzung der stationären Morgenvisite erforderlich. Hieraus ergab sich die Schwierigkeit eine Balance zu finden; einen effektiven Arbeitsablauf zu gewährleisten, aber gleichzeitig auch die Patienteninteressen bei der Visite zu berücksichtigen [3].

Mit dieser prospektiven Studie soll gezeigt werden, entgegen bisheriger Ergebnisse zur Patientenzufriedenheit und Visitenführung [1, 2, 3, 9, 17, 25, 30], ob es dennoch möglich ist, durch CPs, kompetente Visitenbesetzung und Visitengestaltung eine hohe Patientenzufriedenheit zu erreichen.

Die rekrutierten Probanden wurden anhand der Länge ihres stationären Aufenthalts in 2 Gruppen eingeteilt. Vor Studienbeginn wurde ein Beobachtungszeitraum festgelegt. Im Einzelnen sollen folgende Parameter analysiert werden: 
- Begrüßung des Patienten zur Visite (\%)

- Verabschiedung des Patienten am Ende der Visite (\%)

- Körperliche Untersuchung zur Visite (\%)

- Operationserklärung zur Visite (\%)

- Visitenlänge (min)

- Arzt-Patientgespräch (min)

- Arztfragen pro Visite ( $n$ )

- Allgemeine Schmerzbefragung (\%)

- Patientenfragen zur Visite $(\mathrm{n})$

- Patientenerwartung zur Visitenlänge (min)

- Patientenzufriedenheit (\%) 


\section{Material und Methode}

\subsection{Studiendesign}

Die prospektiv angelegte Studie wurde am Universitätsklinikum des Saarlandes in der Klinik für Allgemeine-, Viszeral-, Gefäß- und Kinderchirurgie vom März 2009 bis Juni 2009 durchgeführt. Hierbei wurde eine Station mit einer maximalen Kapazität von 23 Betten, darunter 6 Betten innerhalb einer integrierten Überwachungseinheit (Intermediate Care Unit), mit ausschließlich viszeral- und gefäßchirurgischen Patienten, ausgesucht. In diesem Zeitraum konnten die Angaben von 86 Patienten ausgewertet werden. Die Beobachtungen und Aufzeichnungen wurden durch eine nicht in der Universitätsklinik des Saarlandes tätige approbierte Ärztin durchgeführt.

\subsection{Patientenrekrutierung}

In der Studie wurde ein Beobachtungsfenster vom 1. bis zum 5. postoperativen Tag (POT) und am Entlassungstag (ET) festgelegt. Die Patienten wurden präoperativ entweder am Abend vor der Operation (OP) oder bei Aufnahme am OP-Tag am selbigen über das Projekt informiert. Die Patienten erklärten sich schriftlich mit der Durchführung der Studie einverstanden, welche sich auf morgendliche Visitenbeobachtungen, Tonbandaufnahmen der Visiten und Interviews erstreckte. Das präoperative Interview diente dazu die Patienten zu ihren eventuell zurückliegenden stationären Krankenhausaufenthalten zu befragen.

Den Mitarbeitern der Station wurden keine Informationen über die Beobachtungsparameter und die Fragen in den Interviews gegeben, um einer Verfälschung des Ergebnisses entgegenzuwirken.

Alle Patienten wurden anhand der Länge ihres stationären Aufenthaltes in 2 Gruppen eingeteilt. Gruppe 1 umfasst das Patientenkollektiv mit einer Verweildauer von > 5 (46 Probanden) und Gruppe 2 wird gebildet von Patienten mit $>1$ und $\leq 5$ (40 Probanden) stationären Krankenhaustagen.

Von der zuständigen Ethik-Kommission wurde vor Studienbeginn ein Votum zur Durchführung dieser Studie eingeholt. 


\subsection{Ausschlusskriterien}

Von der Studie ausgeschlossen waren Patienten, die unter Betreuung oder Vormundschaft standen. Auch aus der Studie ausgeschlossen waren Patienten mit psychiatrischen Begleiterkrankungen und Patienten mit postoperativem Durchgangssyndrom.

Die Aufnahmen und Beobachtungen aus der ersten Woche wurden nicht in die Auswertungen einbezogen, um einen Gewöhnungseffekt seitens der Mitarbeiter auf der Station zu erreichen.

\subsection{Präoperative Patientenbefragung}

Die präoperative Patientenbefragung wurde zur Erhebung von Vorerfahrungen zu stationären Aufenthalten und der Erwartungshaltung an Visiten und Ärzten auf Station erhoben. Ebenfalls erfragt wurde die Kenntnis zum Krankheitsbild und der anstehenden OP. Des Weiteren wurden die Patienten auch zu ihrer Schulbildung befragt (Tabelle 1).

Tabelle 1: Präoperative Patientenbefragung bei 86 beobachteten Patienten auf einer chirurgischen Station.

- Ist dies Ihr erster stationärer Krankenhausaufenthalt?

○ Ja

- Nein

- Wie viele Krankenhausaufenthalte gingen dem jetzigen voraus? Anzahl:

- Wie beurteilen Sie die Visitenqualität bei dem/den vorhergehenden stationären Aufenthalt/en?

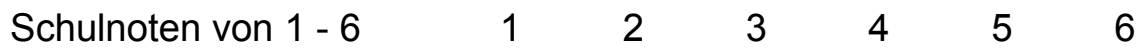

- Fühlen Sie sich ausreichend über Ihr Krankheitsbild informiert?

- Ja

- Nein

- Fühlen Sie sich ausreichend über Ihre Operation informiert?

- Ja

- Nein

- Liegt bei Ihnen ein Tumorleiden vor?

○ Ja

- Nein

- Erwarten Sie, dass Sie von Ihrer Erkrankung geheilt werden?

- Ja

- Nein 
- Wie lange dauert schätzungsweise Ihrer Meinung nach ein durchschnittliches ArztPatientengespräch bei einer Visite? Angabe in min:

- Möchten Sie über alle Befunde ausführlich informiert werden?

○ Ja

○ Nein

- Wie wichtig ist es Ihnen in Entscheidungsprozesse aktiv miteingebunden zu werden?

○ Sehr wichtig

$\circ$ Wichtig

$\circ$ Weniger wichtig

○ Unwichtig

- Haben Sie Angst vor einem Krankenhausaufenthalt?

- Ja

- Nein

- Haben Sie Angst vor Ärzten?

- Ja

- Nein

- Haben Sie Angst vor Operationen?

○ Ja

- Nein

- Haben Sie Angst vor der Visite?

- Ja

- Nein

- $\quad$ Falls ja, warum haben Sie Angst vor der Visite?

○ Erläuterungen nicht verstehen zu können

- Anzahl der anwesenden Personen

- Sonstiges

- Mit wie vielen Personen rechnen Sie bei einer Visite?
- Mehr als 2 Personen
- Mehr als 5 Personen
- Mehr als 10 Personen

- Liegt bei Ihnen eine der folgenden Erkrankungen vor?

○ Alkoholabhängigkeit

- Medikamentenabhängigkeit

○ Drogenabhängigkeit

- Warum haben Sie sich zur Behandlung Ihrer Krankheit für die Universitätsklinik und nicht für ein städtisches Krankenhaus entschieden?
- Empfehlung
- Erfahrung
- Einweisung

- Über welchen Schulabschluss verfügen Sie?

- Kein Schulabschluß

- Hauptschulabschluß

- Realschulabschluß

- Abitur

- Über welche Berufsausbildung verfügen Sie?
- Lehre
- Studium
○ Keine Berufsausbildung 


\subsection{Visitenbeobachtung}

Bei jeder Visite wurde der Visitenbeginn notiert und das Tonbandgerät eingeschaltet.

Es wurde auf Begrüßung, Vorstellung des Arztes, Visitenführung, Durchführung einer körperlichen Untersuchung, Anordnung von Diagnostik und / oder Schmerzmedikation und Verabschiedung geachtet. Die anderen Parameter wie Anzahl der Fragen an den Patienten, Patientenfragen und die Dauer der Visite konnten mittels der Tonbandaufnahme ermittelt werden (Tabelle 2).

Tabelle 2: Visitenbeobachtung und Dokumentation bei 86 beobachteten Patienten vom 1. bis zum 5. postoperativen Tag (POT) und Entlassungstag (ET) auf einer chirurgischen Station.

- Gab es eine Vorbesprechung vor dem Patientenzimmer?

○ Ja

- Nein

- Wurde der Patient zu Beginn der Visite begrüßt?

- Ja

- Nein

- Hat sich der Arzt vorgestellt?

○ Ja

○ Nein

- Liegen Komplikationen vor?

○ Ja

- Nein

- Falls ja, welche Komplikationen liegen bei dem Patienten vor?
○ Wundinfektion
- Harnwegsinfekt
- Pneumonie

- Falls ja, an welchem postoperativen Tag traten die Komplikationen auf? $?^{2-5}$ - Datum:

- Falls ja, wurde dem Patienten die Bedeutung der Komplikation/en erklärt? $?^{2-5}$

○ Ja

- Nein

- Durch welchen Arzt wurde die Visite geführt?

- Oberarzt

- Assistenzarzt

- Oberarzt und Assistenzarzt

- Wurde dem Patienten die durchgeführte OP erklärt? ${ }^{1}$

o Ja

- Nein

- Wurde dem Patienten der Befund erklärt?

○ Ja

- Nein 
- Falls ja, welcher Befund wurde dem Patienten erklärt? $?^{2-5}$

○ OP

- Labor

- Radiologie

- Sonstige

- Wurde weitere Diagnostik angeordnet?

○ Ja

- Nein

- Wurde eine klinische Untersuchung am Patienten durchgeführt?

- Ja

- Nein

- Wurde die klinische Untersuchung vor Anordnung von Diagnostik durchgeführt?

- Ja

- Nein

- Für welchen Tag ist die Entlassung geplant? ${ }^{1}$ POT:

- Visitendauer am Patienten? Angabe in min:

- Direkte Auseinandersetzung mit dem Patienten. Angabe in min:

- Zeitdiskrepanz zwischen Visite und Patientenbefragung. Angabe in min:

- Wurden Fragen an den Patienten gestellt?

○ Ja

- Nein

- Falls ja, wie viele Fragen wurden an den Patienten gestellt? Anzahl:

- Falls ja, wie viele offene Fragen wurden an den Patienten gestellt? Anzahl:

- Falls ja, wie viele geschlossene Fragen wurden an den Patienten gestellt? Anzahl:

- Falls ja, haben mehrere Ärzte Fragen an den Patienten gestellt?

○ Ja

- Nein

- Wurde der Arzt vom Patienten unterbrochen?

- Ja

- Nein

- Wurde der Patient beim Reden unterbrochen?

○ Ja

- Nein

- Gab es im Rahmen der Visite Auseinandersetzungen oder Diskussionen vor dem Patienten?

○ Ja

- Nein

- War/en dem Arzt Vorbefund/e vom Vortag bekannt?

○ Ja

- Nein

- Wurde der Patient nach Schmerzen gefragt?

- Ja

- Nein

- Wurde der Patient gefragt, ob er die Nacht durchschlafen konnte?

- Ja

○ Nein 
- Wurde der Patient nach der Schmerzintensität gefragt? $\begin{array}{lllllllllll}\text { Analoge Schmerzskala von } 0-10 & 1 & 2 & 3 & 4 & 5 & 6 & 7 & 8 & 9 & 10\end{array}$

- Wurde die Schmerzmedikation bei der Visite angepasst?

$$
\text { ○ Ja }
$$

- Nein

- Wurden im Gespräch mit dem Patienten Fachbegriffe verwendet?
○ Ja
- Nein

- Falls ja, wie viele Fachbegriffe wurden verwendet?

Anzahl:

- Falls ja, wurde/n dem Patienten der/die Fachbegriff/e erklärt?

○ Ja

- Wurde Studenten etwas erklärt?
- Ja
- Nein

- Wurden Fragen an die Schwestern gestellt?
- Ja
- Nein

- Wurde mit dem Patienten ein separates Abschlußgespräch vereinbart? ${ }^{\mathrm{ET}}$

○ Ja

- Nein

- Falls ja, wurden dem Patienten ambulante Verhaltensmaßnahmen erläutert? ${ }^{\mathrm{ET}}$
○ Ja
- Nein

- Falls ja, welcher der folgenden Punkte wurde angesprochen? ${ }^{\mathrm{ET}}$

- Medikamenteneinnahme

$$
\begin{aligned}
& \text { - Ja } \\
& \text { - Nein }
\end{aligned}
$$

- Entfernung des Nahtmaterials

$$
\begin{array}{ll}
\text { - Ja } \\
\text { Nein }
\end{array}
$$

○ Körperliche Belastbarkeit

$$
\begin{array}{ll}
\circ & \text { Ja } \\
\circ & \text { Nein }
\end{array}
$$

- Rehabilitationsmaßnahmen

$$
\begin{array}{ll}
\circ & \text { Ja } \\
\circ & \text { Nein }
\end{array}
$$

- Wiedereinstieg ins Berufsleben

$$
\begin{array}{ll}
\circ & \text { Ja } \\
\circ & \text { Nein }
\end{array}
$$

- Folgebehandlung bei Tumorpatienten

$$
\begin{array}{ll}
\circ & \text { Ja } \\
\circ & \text { Nein }
\end{array}
$$

- Stellte der Patient Fragen?

$$
\begin{array}{ll}
\text { - Ja } \\
\circ & \text { Nein }
\end{array}
$$

- Falls ja, wie viele Fragen stellte der Patient?

$$
\text { Anzahl: }
$$

- Gab es am Ende der Visite eine Verabschiedung vom Patienten?

$$
\begin{array}{ll}
\text { - Ja } \\
\text { - Nein }
\end{array}
$$


${ }^{1}$ Frage nur am 1. POT

${ }^{2-5}$ Frage nur am 2. bis 5. POT

ET Frage nur am ET

\subsection{Patienteninterview}

Meist im direkten Anschluss an die Morgenvisite wurde das Interview mit den Patienten durchgeführt. Es wurde Rücksicht auf den Wunsch und den Allgemeinzustand des Patienten genommen, was den Zeitpunkt des Interviews angeht. Der Zeitaufwand betrug ca. 10 min. Die meisten Fragen konnten mit ja/nein beantwortet werden. Es wurden auch wenige Katalogfragen und jeweils 2 freie Fragen an die Patienten gestellt. Zur Ermittlung der Schmerzintensität wurde den Patienten das Instrument der analogen Schmerzskala unter Einbeziehung unserer integrierten Schmerzambulanz vorgestellt und sie wurden gebeten, daran ihr subjektives Schmerzerleben während der zurückliegenden Nacht und während der Visite anzugeben (Tabelle 3).

Tabelle 3: Patientenbefragung und Dokumentation bei 86 beobachteten Patienten vom 1. bis 5. postoperativen Tag (POT) und Entlassungstag (ET) auf einer chirurgischen Station

- Waren Sie mit der Visite zufrieden?

○ Ja

- Nein

- Hat der Arzt Sie zu Beginn der Visite begrüßt?

- Ja

- Nein

- Kannten Sie den Arzt?

- Ja

- Nein

- Falls nicht, hat der Arzt sich vorgestellt?

- Ja

- Nein

- Wurden Sie nach Ihren Schmerzen gefragt?

- Ja

- Nein

- Falls ja, wie stark waren die Schmerzen?

o In der Nacht

- Während der Visite

- Falls ja, hat der Arzt auf die Schmerzäußerung reagiert?

○ Ja

- Nein

- Wurde die Schmerzmedikation angepasst?

- Ja

- Nein 
- Stehen Schmerzspitzen in kausalem Zusammenhang mit folgenden Ereignissen? ${ }^{2 .-}$

- Nahrungsaufnahme

- Verbandswechsel

- Drainagenentfernung

- Mobilisierung

- Stuhlgang (einsetzende Darmperistaltik)

- Lagen bei der heutigen Visite Befund/e vom Vortag vor?
○ Ja
- Nein

- Falls ja, war/en der/die Befund/e dem Arzt bekannt?
- Ja
- Nein

- Falls ja, wurde/n der/die Befund/e vom Arzt erläutert?
- Ja
- Nein

- Falls ja, inwieweit verständlich wurde Ihnen der/die Befund/e erläutert?
○ Verständlich
○ Gut verständlich
○ Nicht verständlich

- Hat der Arzt Ihnen während der Visite weitere Fragen gestellt?
- Ja
○ Nein

- Wie viele Fragen hat der Arzt Ihnen gestellt?

- Weniger als 5

- Mehr als 5

- Haben Ihnen mehrere Ärzte Fragen gestellt?
○ Ja
- Nein

- Falls ja, wie empfanden Sie die Tatsache, dass Sie von mehreren Ärzten befragt wurden?
○ Positiv
- Negativ

- Wurde Ihnen der operative Befund und Verlauf erklärt? ${ }^{1 . \text { POT }}$
- Ja
- Nein

- Falls ja, inwieweit verständlich wurde Ihnen der operative Befund und Verlauf erläutert? ${ }^{1 . ~ P O T}$
○ Verständlich
○ Gut verständlich
○ Nicht verständlich

- Hat der Arzt Ihnen den weiteren Krankheitsverlauf erläutert? ${ }^{1 . \text { POT }}$
- Ja
- Nein

- Falls ja, inwieweit verständlich wurde Ihnen der Krankheitsverlauf erläutert? ${ }^{1 . P O T}$

○ Verständlich

○ Gut verständlich

- Nicht verständlich

- Gab es Auseinandersetzungen oder Diskussionen an Ihrem Krankenbett?
- Ja
- Nein

- Falls ja, hatte die Auseinandersetzung oder Diskussion Sie negativ beeinflusst/verunsichert?

- Ja

- Nein 
- Wurden Sie vom Arzt untersucht?

○ Ja

- Nein

- Falls ja, wurde Ihnen der Befund der klinischen Untersuchung mitgeteilt?

○ Ja

- Nein

- Falls ja, inwieweit verständlich wurde Ihnen der Befund erläutert?

○ Verständlich

○ Gut verständlich

- Nicht verständlich

- Falls ja, wurde bei Innen aufgrund der klinischen Untersuchung weitere Diagnostik angeordnet?

○ Ja

- Nein

- Falls ja, wurde Ihnen der Grund für die Anordnung weiterer Diagnostik erläutert?

○ Ja

- Nein

- Falls ja, inwieweit verständlich wurde Ihnen der Grund für die Anordnung weiterer Diagnostik erläutert?

○ Verständlich

○ Gut verständlich

- Nicht verständlich

- Hatten Sie bei der Visite die Möglichkeit Ihre Beschwerden zu äußern?

○ Ja

- Nein

- Hatten Sie bei der Visite das Gefühl, dass Ihnen Informationen vorenthalten wurden?
○ Ja
- Nein

- Hatten Sie das Gefühl, dass die Ärzte unter Zeitdruck stehen?
○ Ja
- Nein

- Wurden Sie mit Fachbegriffen konfrontiert?
- Ja
- Nein

- Falls ja, wurde/n Ihnen der/die Fachbegriffe erläutert?
○ Ja
- Nein

- Wurde Studenten etwas erklärt?

○ Ja

- Nein

- Falls ja, wie bewerten Sie die Tatsache, dass Studenten etwas erklärt wurde?

- Positiv

- Negativ

- Bekamen Sie Gelegenheit im Rahmen der Visite Fragen zu stellen?

- Ja

- Nein

- Falls ja, wurde/n Ihre Frage/n vom Arzt beantwortet?

○ Ja

- Nein

- Inwieweit verständlich wurde/n die Frage/n vom Arzt beantwortet?

○ Verständlich

○ Gut verständlich

○ Nicht verständlich 
- Wurde Ihnen ein separates Abschlußgespräch angebotent? ${ }^{\mathrm{ET}}$

○ Ja

- Nein

- Wurden Ihnen im Rahmen der Visite ambulante Verhaltensmaßnahmen erläutert? ${ }^{\mathrm{ET}}$

○ Ja

- Nein

- Falls ja, welcher der folgenden Punkte wurde angesprochen? ${ }^{\mathrm{ET}}$

○ Medikamenteneinnahme

○ Ja

- Nein

- Entfernung des Nahtmaterials

○ Ja

- Nein

○ Körperliche Belastbarkeit

○ Ja

- Nein

- Rehabilitationsmaßnahmen

○ Ja

- Nein

- Wiedereinstieg ins Berufsleben

○ Ja

○ Nein

- Bei Tumorpatienten Beginn der Chemotherapie bzw. Bestrahlungstherapie

$$
\begin{array}{ll}
\text { - Ja } \\
\text { - Nein }
\end{array}
$$

- Falls ja, wie beurteilen Sie die Erläuterungen über ambulante

Verhaltensmaßnahmen im Rahmen des Abschlussgespräches? ${ }^{\mathrm{ET}}$

○ Verständlich

- Gut verständlich

○ Nicht verständlich

- Falls ja, sind Fragen hinsichtlich ambulanter Verhaltensmaßnahmen bei Ihnen offen geblieben? $^{\mathrm{ET}}$

- Ja

- Nein

- Hatten Sie Fragen die Sie im Rahmen der Visite nicht gestellt haben?

○ Ja

- Nein

- Warum haben Sie Ihre Fragen nicht gestellt?

$\circ$ Unsicherheit?

- Angst?

○ Vergessen?

- Sonstiges?

- Hat der Arzt sich von Ihnen verabschiedet?
- Ja
○ Nein

- Was hat Ihnen bei der Visite gut gefallen?

Angaben:

- Was hat Ihnen bei der Visite nicht gefallen? Angaben:

\footnotetext{
${ }^{1}$ Frage nur am 1. POT

${ }^{2-5}$ Frage nur am 2. bis 5 . POT

ET Frage nur am ET
} 


\subsection{Auswertung und Statistik}

Die Auswertung der Daten erfolgte im Anschluss an die Visitenbeobachtung und an die Durchführung der Interviews. Die Visitenbeobachtungsdaten wurden ergänzt durch Daten, die durch Auswertung der Tonbandaufnahme erhoben wurden. Zu diesen Parametern zählten die Visitendauer und die Arzt-PatientenAuseinandersetzung die mittels einer Stoppuhr ermittelt wurden sowie Anzahl der Arztfragen differenziert in offene und geschlossene Fragen, Fragen an die Schwestern und Fragen seitens des Patienten. Mittels Antwortmöglichkeiten verständlich, gut verständlich und nicht verständlich wurden die Patienten über die Zufriedenheit zum Inhalten der chirurgischen Visite befragt.

Durchschnittswerte wurden als Mittelwert und Standardfehler angegeben. Die Datenerfassung erfolgte über ein Tabellenkalkulationsprogramm (Excel囚, Microsoft). Die statistische Analyse wurde über zwei verschiedene Softwareprogramme

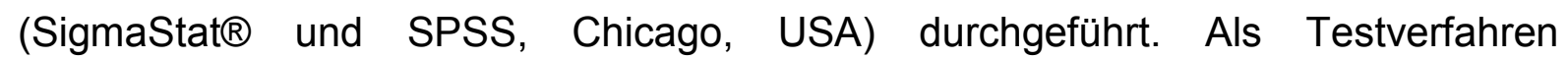
verwendeten wir eine One-way-ANNOVA (numerische Daten), einen Kruskal-WallisRangsummentest (parametrische Daten) oder den Student-T-Test für unverbundene Stichproben, mit einem Signifikanzniveau von $p<0,05$.

\subsection{Ausscheidung und Studienabbrüche}

Retrospektiv musste kein Patient aus der Studie ausgeschlossen werden, da keiner der rekrutierten Patienten postoperativ ein Durchgangssyndrom entwickelt hatte.

Ein Patient aus Gruppe 1 hat nach Erhalt einer infausten Prognose aufgrund des intraopertiven Befundes um Abbruch der Visitenbeobachtung und der Interviews gebeten. Der Abbruch erfolgte am 2. POD nachdem am 1. POD eine Visitenbeobachtung und ein Interview regelrecht laut Studiendesign durchgeführt werden konnte. 


\section{Ergebnisse}

\subsection{Demographische und klinische Daten}

Es wurden 86 Patienten darunter 40 Frauen und 46 Männer mit einem Durchschnittsalter von 56,7 Jahren (Spannweite: 18 - 87 Jahre) in der Studie aufgenommen. Die durchschnittliche Anzahl früherer stationärer Klinikaufenthalte lag bei 5,4 Tagen pro rekrutierten Patienten. 31,4\% unserer Kohorte hatten Abitur oder die mittlere Reife, 66,3 \% absolvierten einen Hauptschulabschluss, während 2,3 \% der Befragten keinen Schulabschluss vorweisen konnten (Tabelle 4).

Tabelle 4: Demographische Angaben zu 86 erfassten Patienten auf einer chirurgischen Station.

\begin{tabular}{l|c|c|c}
\hline Parameter & $\mathbf{n}(\%)$ & MW & SEM \\
\hline Alter & & 56,7 & 15,6 \\
\hline Alter $>60$ & $20(46,5)$ & & \\
\hline Alter $>70$ & $3(3,5)$ & & \\
\hline Alter $>80$ & & & \\
\hline Geschlecht & $40 / 46(46,5 / 53,5)$ & & \\
\hline Weiblich/männlich & $27(31,4)$ & & \\
\hline Schulausbildung/Beruf & $57(66,3)$ & & \\
\hline Abitur oder Realschule & $2(2,3)$ & & \\
\hline Hauptschule & & 5,4 & \\
\hline kein Schulabschluss & $2 / 84(2,3 / 97,7)$ & & \\
\hline vorausgegangene KH Aufenthalte & & & \\
\hline Drogen/Medikamentenabhängigkeit & & & \\
\hline ja/nein & & & \\
\hline
\end{tabular}

MW: Mittelwert

SEM: Standardfehler

In 61,6 \% der Fälle wurde eine maligne und in 38,4 \% der Fälle wurde eine benigne Erkrankung operativ behandelt. Von diesen rekrutierten Patienten mussten 31,4\% auf einer Überwachungseinheit medizinisch versorgt werden. Die Patienten hatten eine durchschnittliche stationäre Verweildauer von 7,2 Tagen, wobei 46,5\% der Patienten weniger als 5 oder genau 5 stationäre Aufenthaltstage benötigten und 53,5 
\% der Patienten einen Krankenhausaufenthalt von mehr als 5 Tagen benötigten. $69,8 \%$ der in dieser Untersuchung erfassten Patienten wurden über unsere Prämedikationsambulanz als CPs erfasst. (Gruppe 1: 60,9 \% und Gruppe 2: 80,0 \%, Tabelle 5).

Tabelle 5: Klinische Angaben zu 86 erfassten Patienten auf einer chirurgischen Station mit integrierter Wachstation (intermediate care (IMC)) unter Einbeziehung von Patientenpfaden (clinical pathways) vom ersten postopertaiven Tag (POT) bis zum Entlassungstag (ET).

\begin{tabular}{lcc}
\hline Parameter & $\mathbf{n}(\%)$ & MW \\
Clinical Pathways & $60(69,8)$ & \\
Diagnose & \\
maligne/benigne & $53 / 33(61,6 / 38,4)$ \\
Therapie & \\
Gefäßeingriffe & $9(10,5)$ \\
Laparotomie & $32(37,2)$ \\
Laparoskopie & $27(31,4)$ \\
Sonstige Eingriffe & $18(20,9)$ \\
Folgeoperationen im gleichen Aufenthalt & $2(2,3)$ \\
Komplikationen & $5(5,8)$ \\
IMC (Im Beobachtunszeitraum 1.- 5. POT) & \\
ja/nein & $27 / 69(31,4 / 68,6)$ \\
Rückverlegung von Normalstation auf IMC & $2(2,3)$ \\
Mittlere Verweildauer (Tage) & \\
Spannweite & $1-38$ \\
Verweildauer $\leq 5$ Tage & $40(46,5)$ \\
Verweildauer $>5$ Tage & $46(53,5)$ \\
\hline
\end{tabular}

MW: Mittelwert

SEM: Standardfehler

\subsection{Präoperative Patientenerwartung}

Die Auswertung der präoperativen Patientenerwartung ergab, dass sich $94,1 \%$ ausreichend über ihr Krankheitsbild und sich 94,9\% ausreichend über die anstehende OP durch das ärztliche Personal aufgeklärt fühlten (Gruppe 1: 97,8 bzw. 
93,5 \% und Gruppe 2: 90,0 bzw. 97,4\%, Zahlen tabellarisch nicht gezeigt). Die Patienten erwarteten eine durchschnittliche Visitenlänge von 4,9 min (Spannweite: 0,75 - 13 min, 4,4 min in Gruppe 1 und 5,3 min in Gruppe 2, Abbildung 1) 

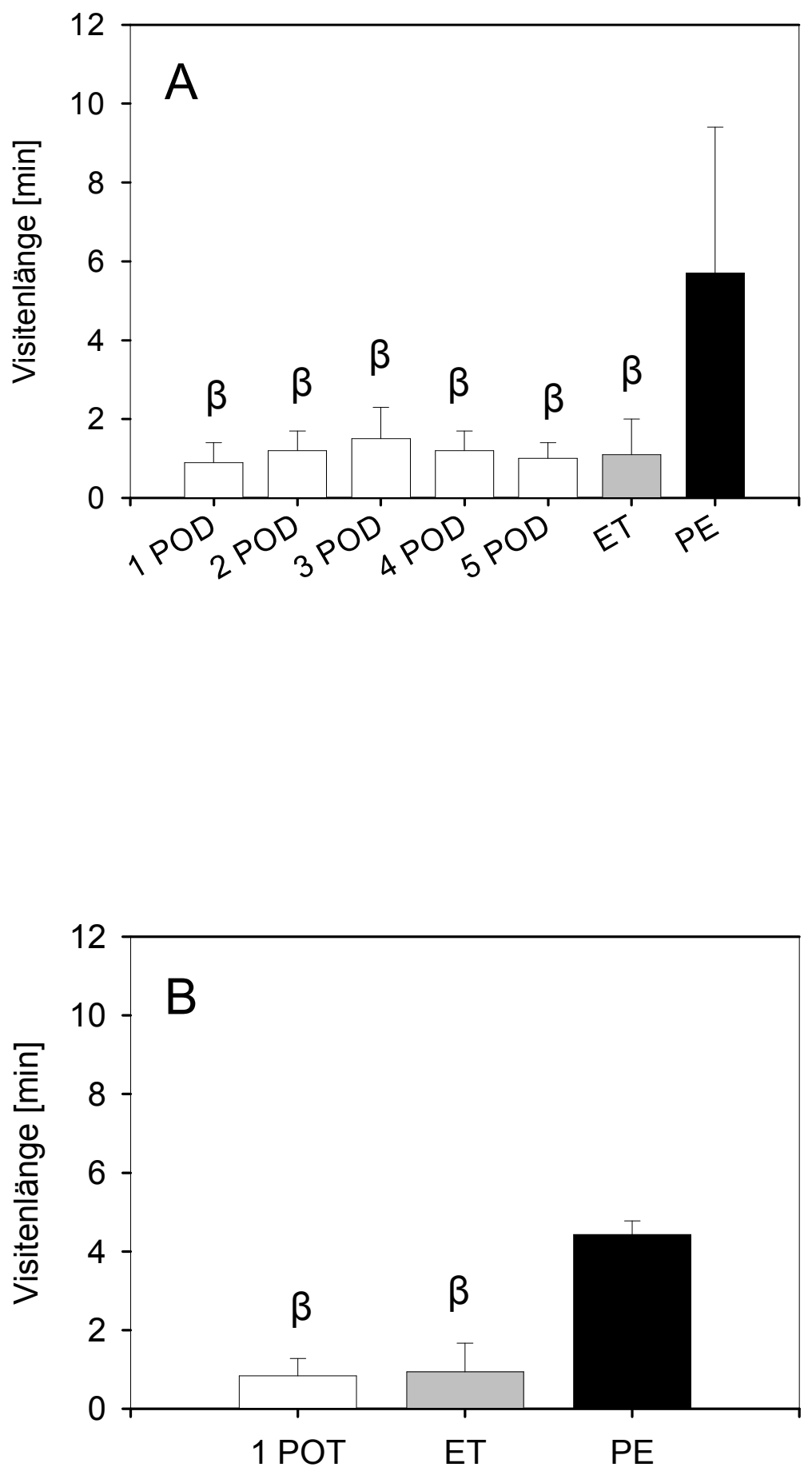

Abbildung 1: Visitenauswertung und Dokumentation bei 86 beobachteten Patienten vom 1. bis zum 5. postoperativen Tag (POT) und Entlassungstag (ET) auf einer chirurgischen Station mit Anbindung einer Überwachungseinheit. Erfassung des Parameters "Visitenlänge" bei Patienten mit stationären Aufenthalt $>5$ Tage $(A)$ und $>1$ bis $\leq 5$ Tage $(B) ;{ }^{\beta} p<0,05$ versus Patientenerwartung (PE). 


\subsection{Visitenbeobachtung und Patientenzufriedenheit}

Eine ärztliche Begrüßung zur Visite erfolgte jeweils in mehr als 95\% zu Beginn des Visitengespräches und in beiden Gruppen. Signifikante Abweichungen konnten in keiner der beiden Gruppen ermittelt werden. (Abbildung 2). 

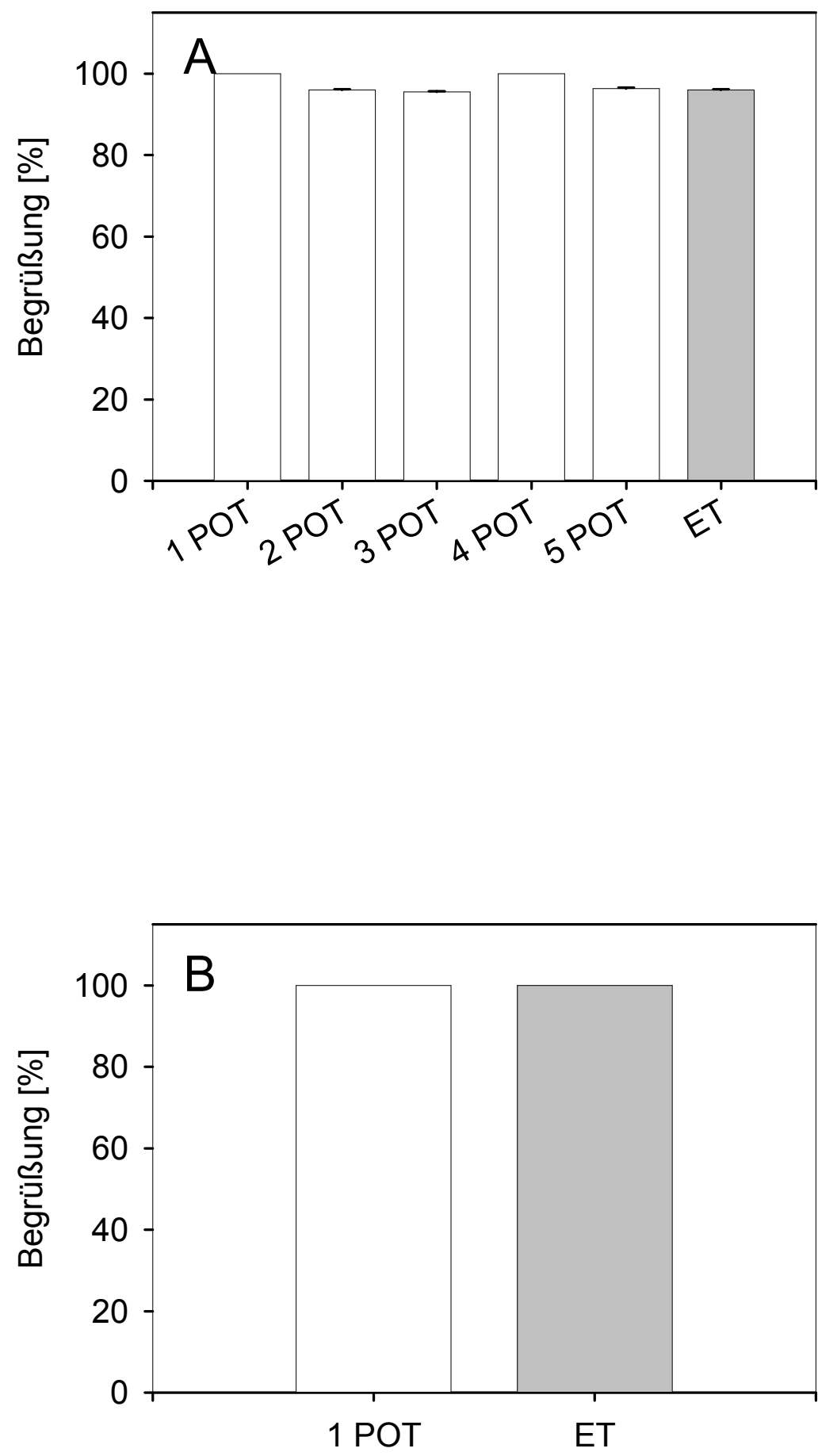

Abbildung 2: Visitenauswertung und Dokumentation bei 86 beobachteten Patienten vom 1. bis zum 5. postoperativen Tag (POT) und Entlassungstag (ET) auf einer chirurgischen Station mit Anbindung einer Überwachungseinheit. Erfassung des Parameters „Begrüßung der Ärzte zur Visite" bei Patienten mit stationären Aufenthalt $>5$ (A) und $>1$ bis $\leq 5$ Tage $(B)$. 
Allerdings gab es große Schwankungen hinsichtlich der ärztlichen Verabschiedung vom Patienten (Spannweite Gruppen 1: 32 \% (2. POT) bis 76 \% (ET), Spannweite Gruppe 2: 63,6 bis 86,7 \%). Hierbei zeigte sich ein signifikanter Unterschied in unserer Gruppe 1 am 2. POT versus 4. POT und versus ET $(p<0,05$, Abbildung 3). 

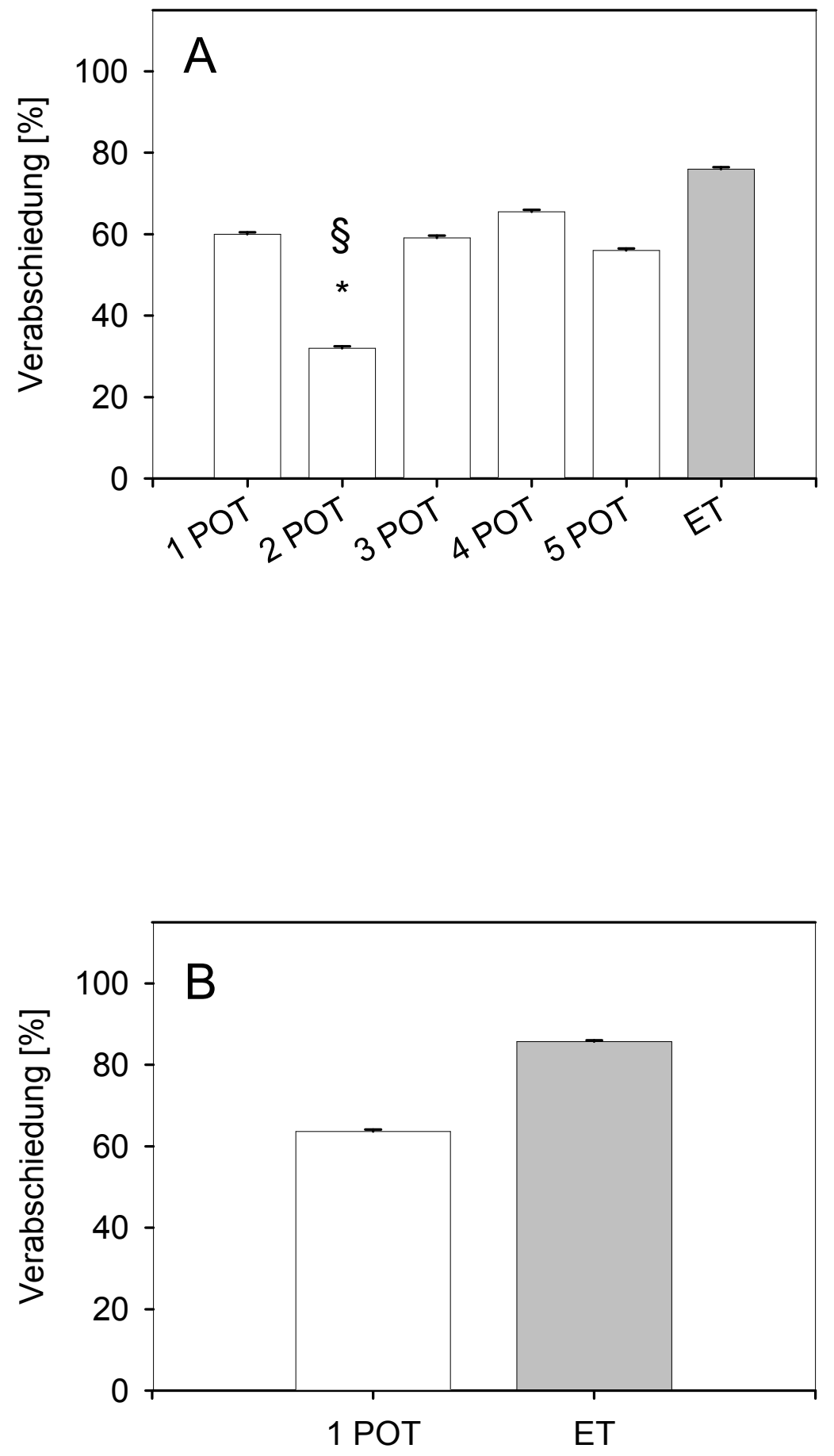

Abbildung 3: Visitenauswertung und Dokumentation bei 86 beobachteten Patienten vom 1. bis zum 5. postoperativen Tag (POT) und Entlassungstag (ET) auf einer chirurgischen Station mit Anbindung einer Überwachungseinheit. Erfassung des Parameters "Verabschiedung der Ärzte vom Patienten am Ende der Visite" bei Patienten mit stationären Aufenthalt $>5$ (A) und $>1$ bis $\leq 5$ Tage (B); ${ }^{*} p<0,05$ versus $\mathrm{ET},{ }_{\mathrm{p}}<0,05$ versus 4 . POT. 
Am 3. POT wurde eine körperliche Untersuchung innerhalb der Gruppe 1 mit $77,3 \%$ am häufigsten durchgeführt ( $p<0,05$ versus 5 . POT), während in Gruppe 2 am ET bei $63,0 \%$ der höchste Wert ermittelt werden konnte. Der geringste Wert an körperlichen Untersuchungen erfolgte am 5. POT in Gruppe 1 mit 32,1 \% (Abbildung 4). 

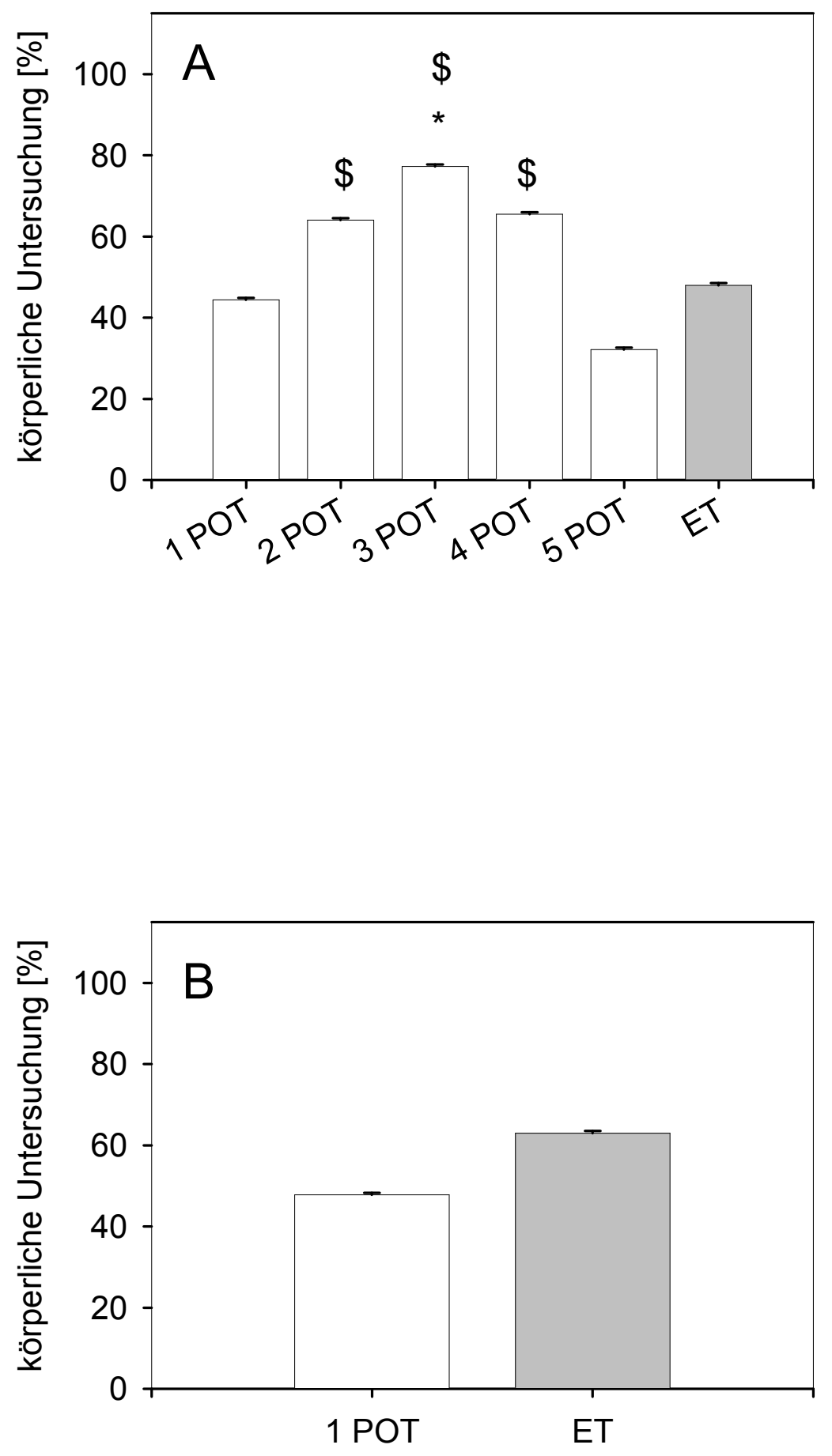

Abbildung 4: Visitenauswertung und Dokumentation bei 86 beobachteten Patienten vom 1. bis zum 5. postoperativen Tag (POT) und Entlassungstag (ET) auf einer chirurgischen Station mit Anbindung einer Überwachungseinheit. Erfassung des Parameters "Körperliche Untersuchung" bei Patienten mit stationären Aufenthalt $>5$ (A) und $>1$ bis $\leq 5$ Tage (B); ${ }^{*} p<0,05$ versus ET und ${ }^{\$} p<0,05$ versus 5. POT. 
Eine genaue Erklärung über den operativen Verlauf wurde signifikant häufiger am 1. POT in 55,6 \% der Fälle in Gruppe 1 durchgeführt ( $p<0,05$ versus 2. - 5 . POT). In 56,5\% der Visitenbeobachtungen wurde am 1. POT in Gruppe 2 dem Patienten Informationen zum intraoperativen Verlauf vermittelt (Abbildung 5). 

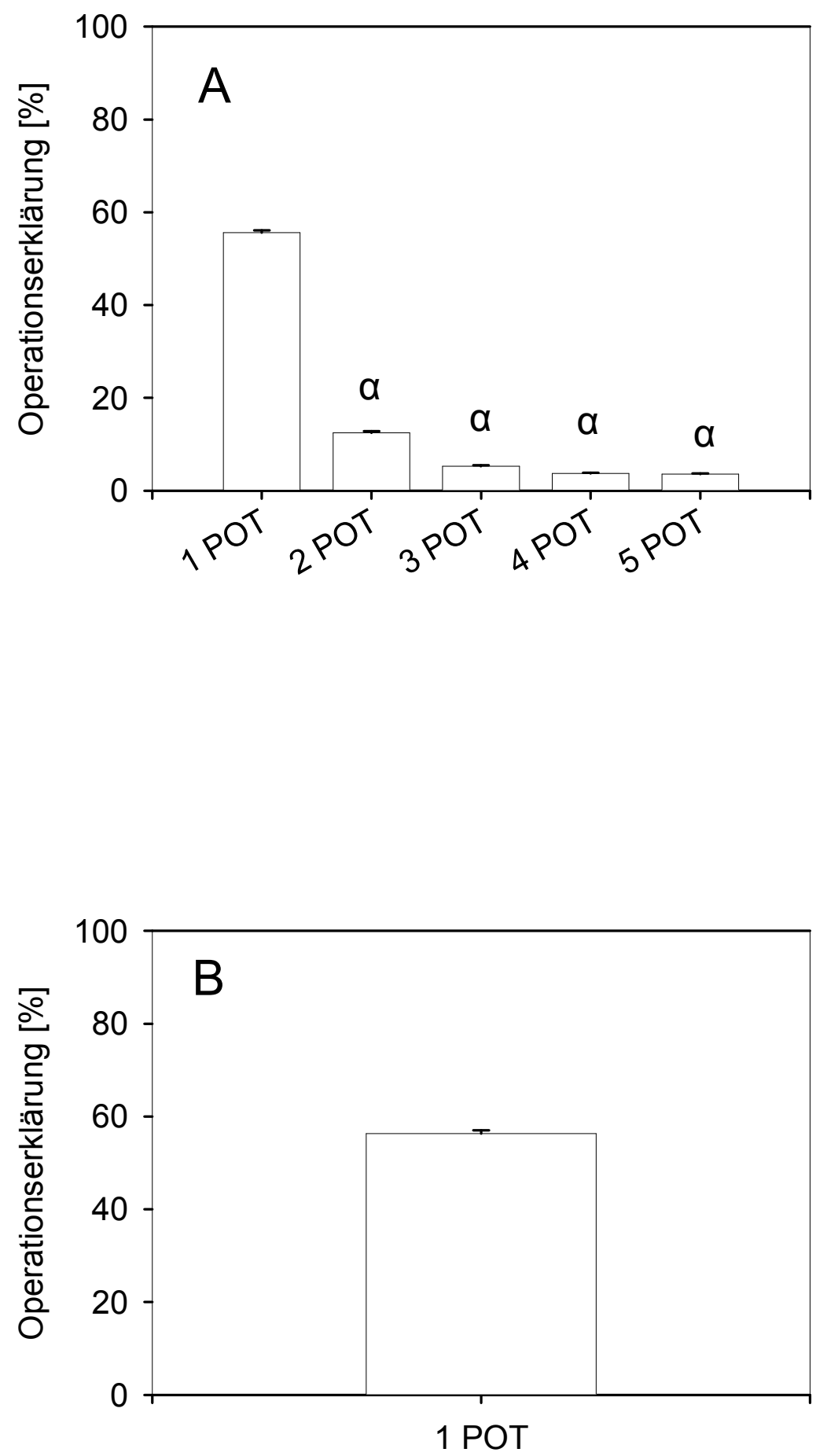

Abbildung 5: Visitenauswertung und Dokumentation bei 86 beobachteten Patienten vom 1. bis zum 5. postoperativen Tag (POT) und Entlassungstag (ET) auf einer chirurgischen Station mit Anbindung einer Überwachungseinheit. Erfassung des Parameters „Operationserklärung" bei Patienten mit stationären Aufenthalt $>5(\mathrm{~A})$, und $>1$ bis $\leq 5$ Tage $(B) ;{ }^{a}<0,05$ versus 1 . POT. 
Durchschnittlich dauerten die Visiten innerhalb der Gruppe 1 am 3. POT 1,5 min und am ET 1,1 min am längsten. Die präoperative PE war allerdings mit 5,3 min signifikant höher ( $p<0,05$ versus 1. - 5. POT und ET, Abbildung 1). In Gruppe 2 belief sich die Visitenlänge auf $0,8 \mathrm{~min}$ am 1. POT und auf $0,9 \mathrm{~min}$ am ET bei erwarteten 4,4 min.

Das reine Arzt-Patientengespräch zur Visite war in Gruppe 1 am 1. POT mit 0,33 min signifikant niedriger als am ET mit 0,93 min. In Gruppe 2 gab es am 1. POT mit 0,49 min und am ET mit 0,68 min keinen signifikanten Unterschied (Abbildung 6). Das Verhältnis Visitenlänge zur Arzt-Patientgespräch zeigte eine Spannweite von 1:0,36 (1. POT) bis 1:0,81 (ET) in Gruppe 1 vergleichbar mit 1:0,58 bis 1:0,91 in Gruppe 2 (Werte graphisch nicht dargestellt). 

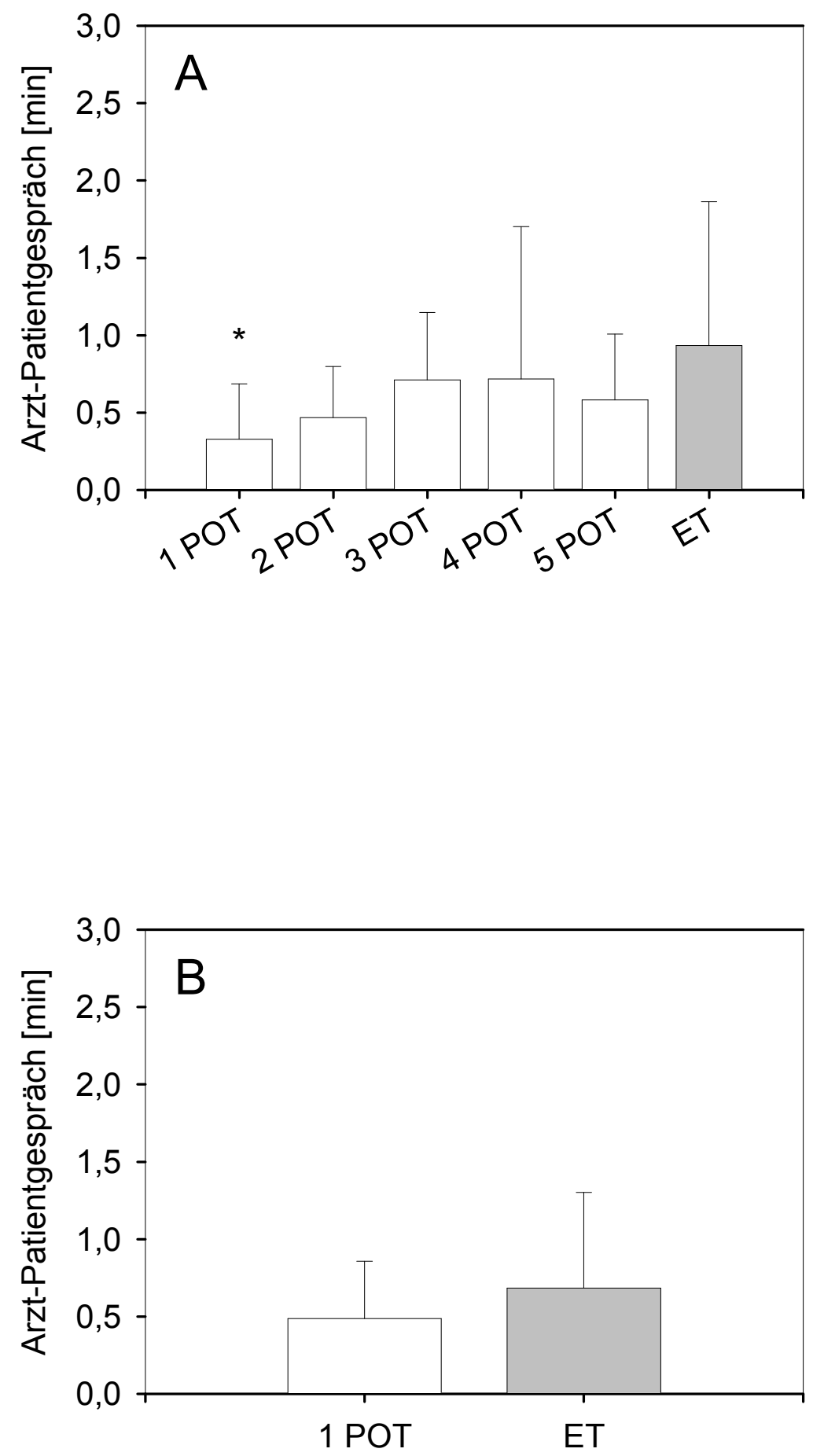

Abbildung 6: Visitenauswertung und Dokumentation bei 86 beobachteten Patienten vom 1. bis zum 5. postoperativen Tag (POT) und Entlassungstag (ET) auf einer chirurgischen Station mit Anbindung einer Überwachungseinheit. Erfassung des Parameters „Arzt-Patientgespräch“ bei Patienten mit stationären Aufenthalt $>5(\mathrm{~A})$, und $>1$ bis $\leq 5$ Tage $(B) ; p<0,05$ versus $E T$. 
Die meisten ärztlichen Fragen an die Patienten zur Visite wurden am 3. POT (durchschnittlich 3,4 Fragen) in der Gruppe 1 ohne signifikanten Unterschied gestellt. An den anderen Tagen im Beobachtungsfenster erhoben die Ärzte am 1. und 2. POT durchschnittlich 2 Fragen und am 4. bis 5. POT und am ET mit 2,4 bis 2,5 annähernd gleiche Anzahl an Fragen an die Patienten. Verglichen mit Gruppe 1 wurde in Gruppe 2 an beiden Beobachtungstagen eine weniger intensive ärztliche Befragung mit 1,5 versus 1,9 Fragen durchgeführt (Abbildung 7). 

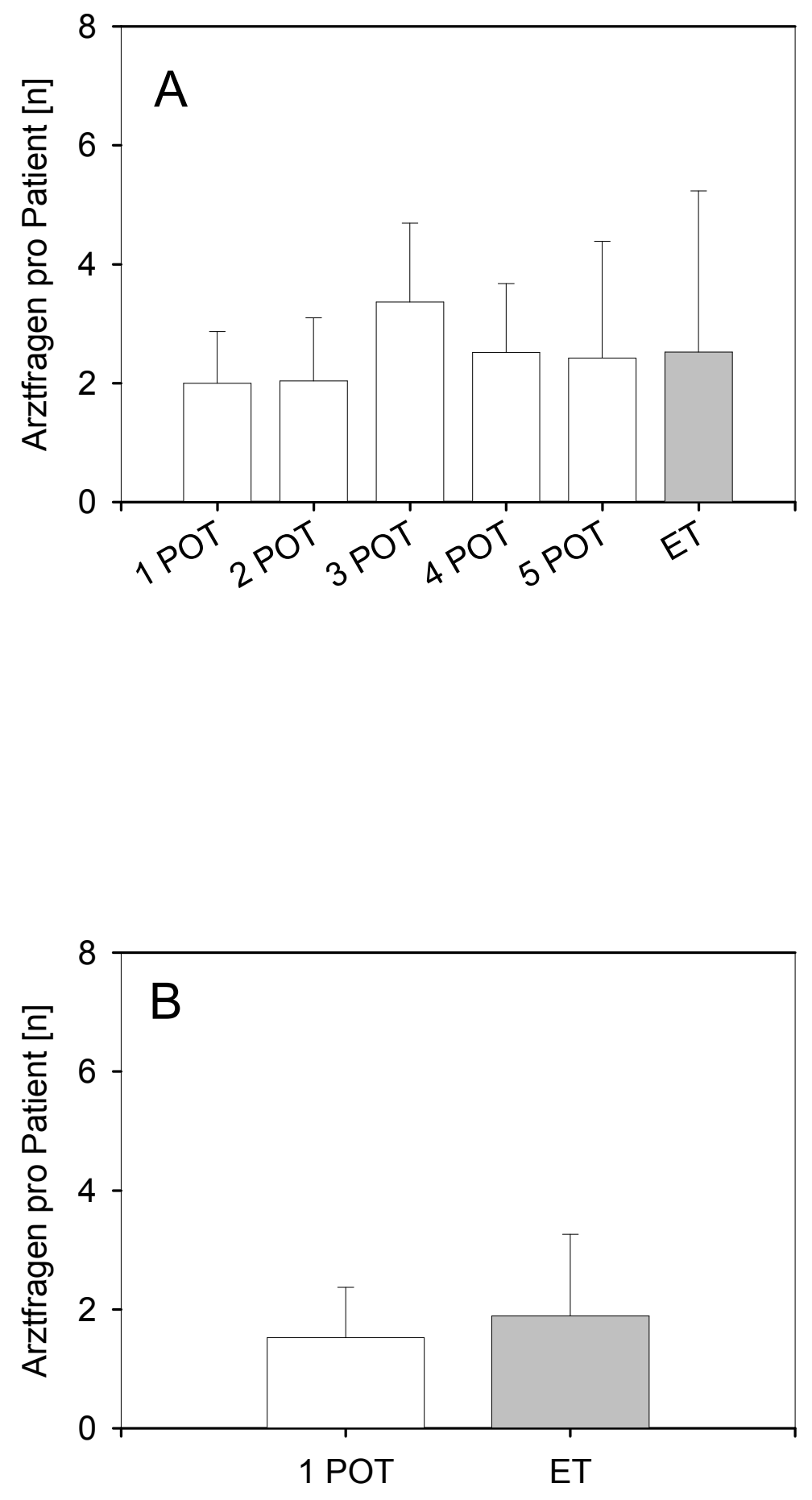

Abbildung 7: Visitenauswertung und Dokumentation bei 86 beobachteten Patienten vom 1. bis zum 5. postoperativen Tag (POT) und Entlassungstag (ET) auf einer chirurgischen Station mit Anbindung einer Überwachungseinheit. Erfassung des Parameters „Arztfragen pro Visite“ bei Patienten mit stationären Aufenthalt $>5$ (A) und $>1$ bis $\leq 5$ Tage $(B)$. 
Bei der Auswertung des Frageninhaltes zeigte sich, dass am 1. POT in 77,8 $\%$, respektive $73,9 \%$ der Fälle in Gruppe 1 und 2 nach postoperativen Schmerzen gefragt wurde. Signifikante Unterschiede konnten in keiner der beiden Gruppen ermittelt werden (Abbildung 8). 

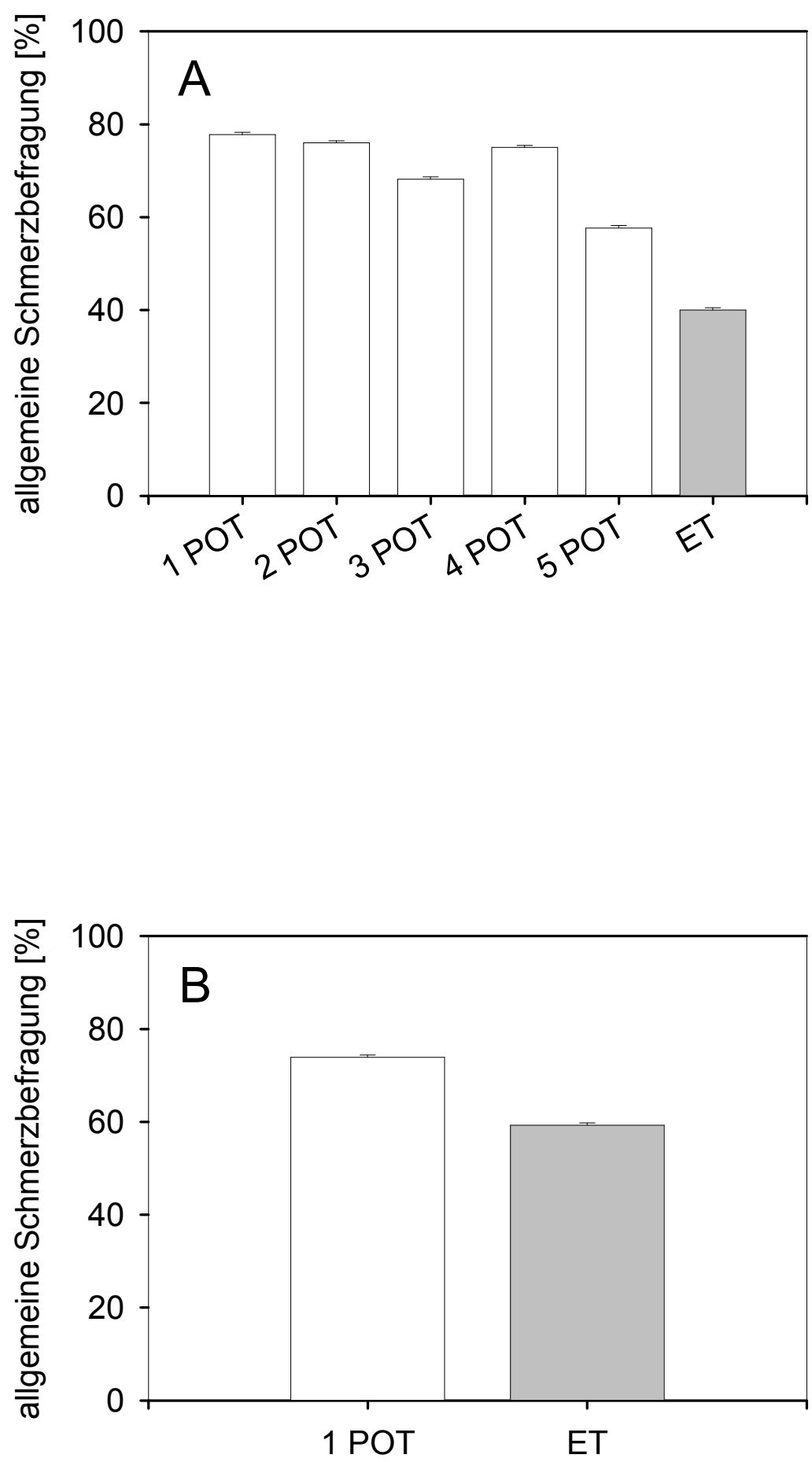

Abbildung 8: Visitenauswertung und Dokumentation bei 86 beobachteten Patienten vom 1. bis zum 5. postoperativen Tag (POT) und Entlassungstag (ET) auf einer chirurgischen Station mit Anbindung einer Überwachungseinheit. Erfassung des Parameters "allgemeine Schmerzbefragung" bei Patienten mit stationären Aufenthalt $>5$ (A) und $>1$ bis $\leq 5$ Tage $(B)$. 
Der Informationsbedarf der Patienten am ET in Gruppe 1 war signifikant im Vergleich zu den Tagen 2. bis 5. POT mit durchschnittlich 1,5 Fragen pro Visite erhöht. In Gruppe 2 konnte bei der Auswertung kein signifikanter Unterschied ermittelt werden (Abbildung 9). 

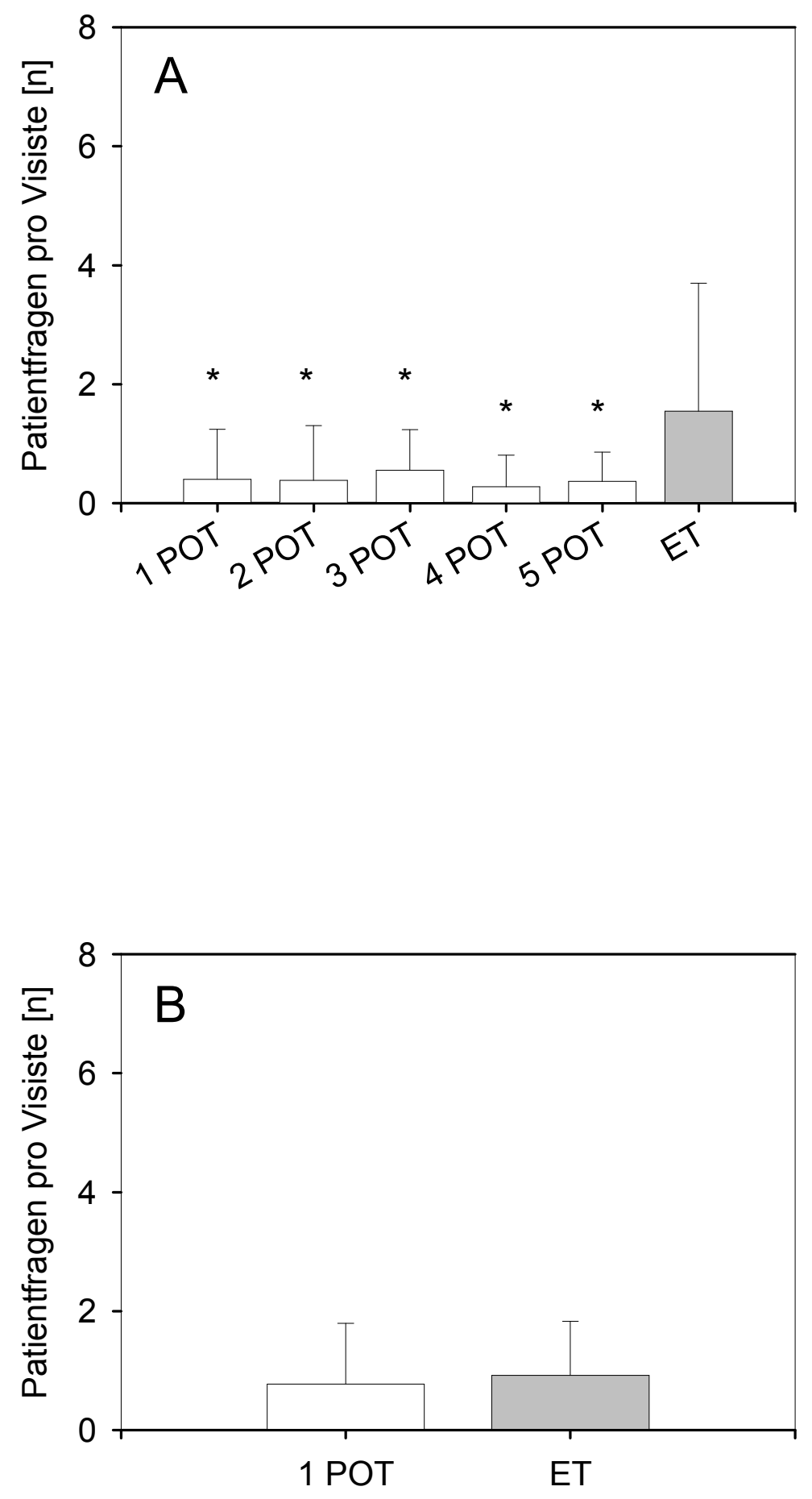

Abbildung 9: Visitenauswertung und Dokumentation bei 86 beobachteten Patienten vom 1. bis zum 5. postoperativen Tag (POT) und Entlassungstag (ET) auf einer chirurgischen Station mit Anbindung einer Überwachungseinheit. Erfassung des Parameters "Patientenfragen pro Visite" bei Patienten mit stationären Aufenthalt $>5$ (A), und $>1$ bis $\leq 5$ Tage (B); $p<0,05$ versus ET 
In beiden Gruppen konnte eine Patientenzufriedenheit stets von mehr als 80 $\%$ erreicht werden. Bei der Auswertung konnten in beiden Gruppen keine signifikanten Unterschiede ermittelt werden (Abbildung 10). 

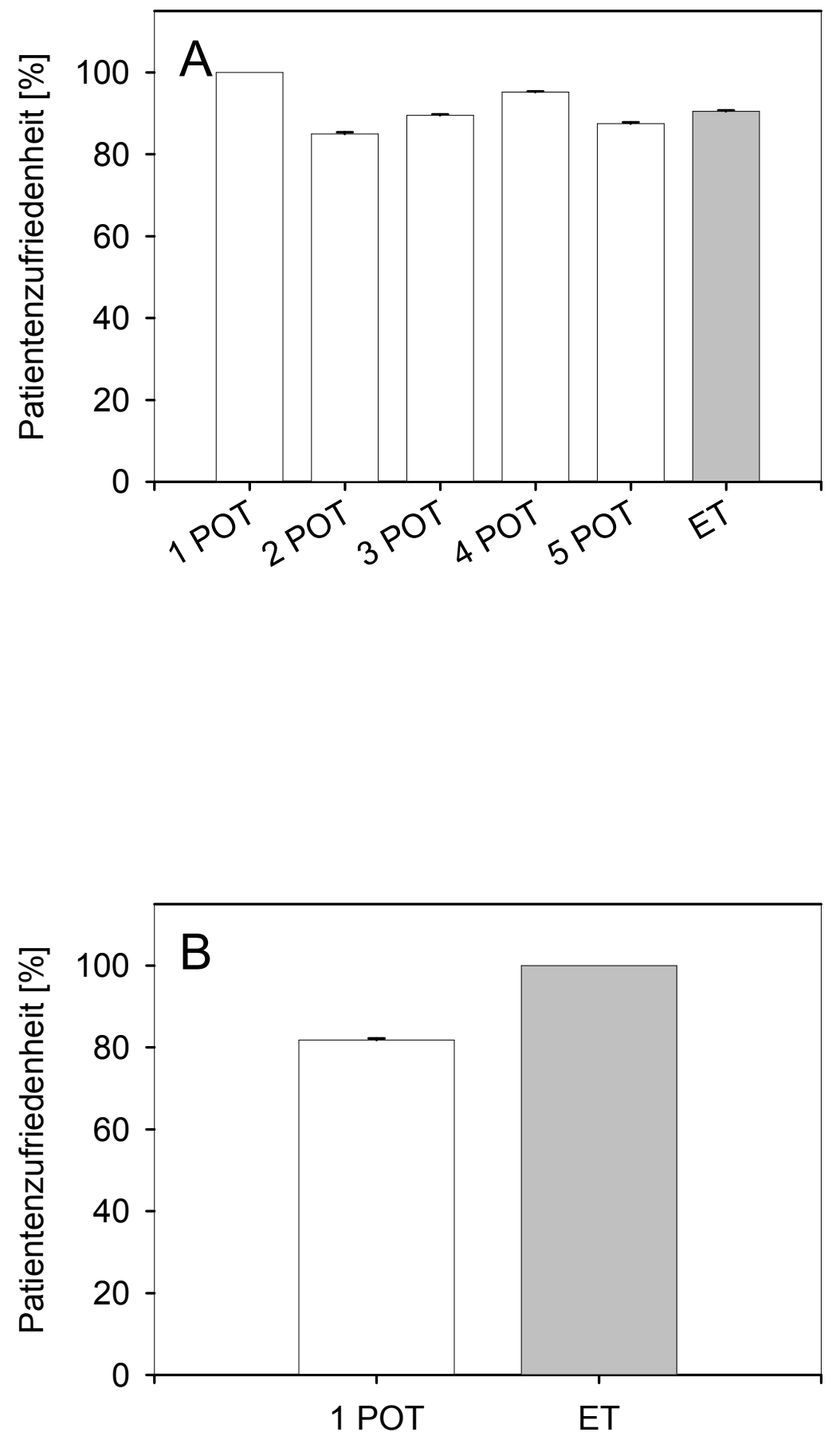

Abbildung 10: Visitenauswertung und Dokumentation bei 86 beobachteten Patienten vom 1. bis zum 5. postoperativen Tag (POT) und Entlassungstag (ET) auf einer chirurgischen Station mit Anbindung einer Überwachungseinheit. Erfassung des Parameters „Patientenzufriedenheit" bei Patienten mit stationären Aufenthalt $>5$ (A) und $>1$ bis $\leq 5$ Tage (B). 


\subsection{Patientenresonanzen zur Visite}

Am Ende eines jeden Patienteninterviews wurde den Patienten mit der Frage „Was hat Ihnen heute bei der Visite gut gefallen?“ bzw. „Was hat Ihnen heute bei der Visite nicht gefallen?" eine Plattform geboten ihre subjektiven Eindrücke zur Visite zu formulieren. An positiven Resonanzen wurden von Patientenseite eine angenehme Gesprächsführung und -atmosphäre, Freundlichkeit und Aufmunterung angegeben. Als negative Resonanz zur Visite gaben die Patienten die große Anzahl an teilnehmenden Personen zur Visite an.

\subsection{Geschlechtsvergleich}

Bezüglich der Visitenlänge konnte sowohl am 3. als auch am 4. POT zwischen den Geschlechtern je eine Differenz der von 0,2 min ermittelt werden. Am 3. POT betrug die Visitenlänge bei Frauen durchschnittlich 1,6 min verglichen mit 1,4 min bei Männern. Am 4. POT wurden Frauen durchschnittlich 1,4 min visitiert, während bei Männern die Visitenlänge 1,2 min betrug. Bezüglich der Visitenlänge konnte bei den weiblichen Probandinnen zwischen dem 1. und 3. POT eine statistisch signifikante Abweichung mit $p<0,05$ ermittelt werden. Bei Auswertung der Daten der rekrutierten männlichen Probanden konnte keine Signifikanz ermittelt werden.

Im Rahmen der Visiten wurde seitens der Ärzte am 3. POT bei beiden Geschlechtern die ausgiebigste Befragung durchgeführt. Dabei wurden an die Probandinnen am 3. POT durchschnittlich 3,3 Fragen gestellt verglichen mit den Probanden, bei denen an dem selben Beobachtungstag 3,2 Fragen gestellt wurden. Bei den weiblichen Studienteilnehmerinnen konnten am 3. und 5. versus ET, am 3. und 5. POT versus 1. POT sowie am 3. und 5. POT versus 2. POT eine signifikante Abweichung von $p<0,05$ ermittelt werden. Die Auswertung der ärztlichen Fragen an die männlichen Probanden ergab keine statistische Signifikanz.

Bei beiden Geschlechtern konnten am 2. und 4. POT ein geringere Informationsbedarf verglichen mit den anderen Beobachtungstagen ermittelt werden. Am Entlassungstag bestand bei beiden Geschlechtern mit 1,1 bei Frauen versus 0,7 Fragen bei Männern der höchste Informationsbedarf. Bei den männlichen 
Probanden konnte ein signifikante Unterschied bezüglich des Informationsbedarf zwischen dem 4. und ET ermittelt werden (Abbildung 6).

Tabelle 6: Geschlechtsvergleich von 86 erfassten Patienten auf einer chirurgischen Station mit integrierter Wachstation (intermediate care (IMC)) unter Einbeziehung von Patientenpfaden (clinical pathways) vom ersten postoperativen Tag (POT) bis zum Entlassungstag (ET).

Frauen

\begin{tabular}{l|c|c|c|c|c|c}
\hline POT & 1 & 2 & 3 & 4 & 5 & $\mathrm{ET}$ \\
\hline Visitenlänge [min] (MW \pm SEM) & 0,9 & 1,2 & 1,6 & 1,4 & 1,0 & 1,0 \\
& $\pm 0,1$ & $\pm 0,1$ & $\pm 0,2$ & $\pm 0,2$ & $\pm 0,1$ & $\pm 0,2$ \\
\hline Arztfrage pro Patient (n) & 1,8 & 1,9 & $3,3^{*} \propto$ & 2,8 & $3,5^{*} \alpha$ & 1,7 \\
\hline Patientenfrage pro Visite (n) & 0,7 & 0,3 & 0,6 & 0,3 & 1,1 & 1,1 \\
\hline
\end{tabular}

Männer

\begin{tabular}{l|c|c|c|c|c|c}
\hline POT & 1 & 2 & 3 & 4 & 5 & ET \\
\hline Visitenlänge [min] (MW \pm SEM) & 0,9 & 1,2 & 1,4 & 1,2 & 1,0 & 1,0 \\
& $\pm 0,2$ & $\pm 0,1$ & $\pm 0,1$ & $\pm 0,2$ & $\pm 0,1$ & $\pm 0,2$ \\
\hline Arztfrage pro Patient (n) & 1,5 & 2,1 & 3,2 & 2,2 & 1,7 & 2,6 \\
\hline Patientenfrage pro Visite (n) & 0,4 & 0,4 & 0,5 & 0,2 & 0,4 & $0,7^{\S}$ \\
\hline
\end{tabular}

MW: Mittelwert

SEM: Standardfehler

POT: postoperativer Tag

ET: Entlassungstag

$\S_{p}<0,05$ versus 4 . POT

* $p<0,05$ versus ET

$a_{p}<0,05$ versus 1 . POT

$£_{p}<0,05$ versus 2. POT 


\section{Diskussion}

\subsection{Allgemeines}

Die Einhaltung des Arbeitszeitgesetzes erforderte seit Januar 2009 drastische Strukturänderungen innerhalb unserer chirurgischen Abteilung. Die Visitendauer wurde als eine der Maßnahmen zur Einhaltung des Arbeitszeitgesetzes von 45 auf 30 min gekürzt.

Einzelne Stationsoberärzte wurden hierbei in den täglichen Visitenablauf fest eingebunden. Die erhobenen Daten aus unserer prospektiven Studie zur Erfassung der Patientenzufriedenheit nach drastischer Umstrukturierung der Visiten konnte zeigen, dass trotz deutlicher Kürzungen der Visitenzeiten es dennoch möglich ist, eine hohe Patientenzufriedenheit auf einer chirurgischen Station durch Visitenbesetzung mit Entscheidungsträgern und der routinemäßigen Eingliederung der Patienten in CPs, zu gewährleisten.

Entgegen bisheriger Erkenntnisse auf dem Gebiet der Visitenforschung war die Visitendauer in der untersuchten Kohorte deutlich kürzer. Eine ausgiebige Literaturrecherche ergab, dass andere Studien zur Evaluation von Stationsvisiten deutlich längere Visitenzeiten aufwiesen. In der Arbeit von Siegrist, in der die Visitenlänge zwischen internistisch geführten Stationen und chirurgisch geführten Stationen miteinander verglichen wurden, und eine Differenz von einer min $(2,7$ versus 1,7 $\mathrm{min}$ ) ermittelt wurde, übertrifft die chirurgische Visitendauer mit 1,7 min alle evaluierten durchschnittlichen Visitenlängen in dieser Arbeit [29].

Bei dem Vergleich der Ergebnisse zur Visitenlänge in anderen Fachdisziplinen konnte eine Spannweite von 3 bis 11 min angegeben werden. Nordmeyer ermittelte eine durchschnittliche Visitendauer von 3,5 min in einer Internistischen Klinik mit Schwerpunkt Rheumatologie, Papesdorf ermittelte in der Abteilung für Ophthalmologie des Universitätsklinikums Greifswald eine durchschnittliche Visitendauer von 3,48 min, Raspe und Nordmeyer ermittelten ebenfalls eine Visitendauer von $3 \mathrm{~min}$ bei internistischen Visiten. Mit $5 \mathrm{~min}$ belief sich die durchschnittliche Visitendauer auf einer internistisch geführten Intensivstation in der Arbeit von Friesdorf, noch länger mit 6,5 min betrug die Visite pro Patient auf der internistisch-psychosomatischen Station in der Evaluation von Westphal und Köhle. 
Die ausführlichste Visite wurde in der Arbeit von Probst mit $11 \mathrm{~min}$ im Fachbereich der psychosomatischen Medizin ermittelt [8, 16, 17, 18, 19].

Haferlach bestimmte in seiner Arbeit einen Faktor von 1,9-3,5 der die Überschätzung der Patienten zwischen geschätzter und objektiver Dauer der Visite, deckungsgleich zu diesen Ergebnissen, belegt [10]. Auch Middeke bezeichnete in seiner Arbeit die Dauer der Visite als eher zweitrangig. Hierbei wurde der Inhalt einer Visite weitaus höher bewertet [15].

In der deskriptiv durchgeführten Studie von Nordmeyer wurde der Redeanteil vom ärztlichen Personal und den Patienten ausgewertet und ein Verhältnis von 63 zu $37 \%$ festgestellt. Ergänzend ermittelte Raspe dass ca. 2/3 aller Gesprächsinitiativen vom Arzt ausgehen und nur 1/5 vom Patienten. Von diesem beschriebenen $1 / 5$ Gesprächsinitiative seitens des Patienten resultieren $80 \%$ als Reaktion auf Gesprächsimpulse von Ärzten bzw. Schwestern [21].

Des Weiteren wurde in diesen beiden oben genannten Arbeiten auch das Fragenverhalten zur Visite beleuchtet, welches auch ein untersuchter Parameter in dieser Arbeit darstellte. Bei Nordmeyer zeigte sich in der Auswertung ein mit $82 \%$ überwiegender Fragenanteil verglichen mit 18 \% Fragen von Patienten Seite [16].

Die Zahl der ärztlichen Fragen zur Visite zeigte eine Spannweite von 6 bis 11. Durchschnittlich erhoben die Ärzte in der Studie von Nordmeyer 11 Fragen pro Visite während die Patienten sich mit 2 Fragen ins Visitengespräch einbrachten [16]. Geisler zeigte in seiner Arbeit das Verhältnis ärztlicher offener zu geschlossener Fragen mit 1:10 auf, ein Verhältnis das sich in dieser Studie als weniger ausgeprägt darstellte [8].

Die reine Auseinandersetzung mit dem Patienten belief sich deckungsgleich zu diesen Ergebnissen von Haferlach auf 40-60 Sekunden [10].

Die von den meisten Patienten präferierte passive Rolle in der Interaktion mit dem ärztlichen Personal beleuchtete Garcia-Gonzalez et al [7]. Als Ausdruck dieser eher passiven Rolle zeigte sich das in den vorliegenden Arbeiten übereinstimmend ermittelte geringe Fragennivea von Seiten der Patienten mit der Spannweite 1 bis 2 Fragen pro Visitengespräch und Patient [8, 15, 16, 17, 18]. Verglichen mit den Ergebnissen dieser Studie lag die durchschnittliche Anzahl der Patientenfragen im Rahmen des Visitengespräches in Gruppe 1 vom 1. bis 5. POT unter den Ergebnissen der beiden oben erwähnten Studien. In Gruppe 1 am ET und im 
gesamten Beobachtungszeitraum von Gruppe 2 beliefen sich die ermittelten Frageninitiativen von Seiten der Patienten im Bereich von 1 bis 2 Fragen zur Visite. Die Arbeit von Haferlach zeigte bei Männern signifikant mehr Initiative in Form von Fragen zur Visite, während in Visitengesprächen mit Frauen von ärztlicher Seite signifikant mehr offene Fragen formuliert wurden. Diese beiden geschlechtsspezifischen Unterschiede zur Visite konnte man in dieser Arbeit nicht bestätigen [10].

\subsection{Umstrukturierung der Visitenführung}

Auch bei kurzer Verweildauer der Patienten in unserer Klinik entstanden positive Bewertungen. Ausgehend davon, dass es gerade die Visiten sind, welche die häufigsten Kontakte zwischen Patienten und Krankenhauspersonal sind, hat es entscheidenden Anteil daran, wie der Patient letztlich seinen Krankenhausaufenthalt bewertet und weiter kommuniziert. Die Besetzung von Entscheidungsträgern zu den Routinevisiten wurde stellenweise in bisherigen Arbeiten als kritisch bewertet [5, 8]. Engelhardt beurteilte sogenannte „große Visiten“ im Sinne einer Oberarzt- und Chefarztbesetzung $z u$ den Visiten als besonders kritisch mit negativer Patientenzufriedenheit [5]. Im Gegenteil konnte die hier aufgeführte Studie zeigen, dass gerade die Besetzung der Visiten mit Oberärzten den Patienten das Gefühl von Sicherheit und Kompetenz gab. Hierbei war die unmittelbare Entscheidung zur weiterführenden Diagnostik oder Interpretation der bisherigen Ergebnisse und Erkenntnisse mit konkreten Aussagen an chirurgische Patienten direkt am Patientenbett ohne weitere zeitliche Verzögerung maßgebend.

\subsection{Clinical Pathways}

Von der Konzeption her scheinen solche standardisierten, evidenzbasierten CPs, auch Pfade genannt, ebenso einleuchtend wie überzeugend: Ausgehend von der Perspektive des Patienten und seinen Bedürfnissen erlauben sie eine systematische Ausschöpfung von Effektivitäts- und Effizienzpotenzialen über alle Stufen der Behandlung hinweg - von der Aufnahme über die Diagnostik, Anästhesie, Operation 
und Pflege bis hin zur Entlassung [26]. Zugleich ermöglichen sie die Abkehr von oft beklagten dogmatischen Arbeitsaufteilungen der Berufsgruppen und Funktionen und ermöglichen eine sorgsam eingespielte Zusammenarbeit in interdisziplinär besetzten Teams, bestehend aus Oberärzten, Assistenzärzten, Pflegern und medizinischtechnischen Spezialisten [26]. Durch die CPs vermittelten Leitlinien konnte die Anzahl an körperlichen Untersuchungen konzentriert werden. Die Homburger CPsLeitlinien beinhalten den automatisierten Ablauf von diagnostischen Maßnahmen (Wunddokumentation, Drainagesekretbestimmung, Routinelabor, radiologischer Breischluck, etc.) zu den jeweiligen POT, welche das Pflegepersonal mit einbeziehen, wodurch das ärztliche Personal entlastet werden konnte. Eine erhöhte Inzidenz an Komplikationen konnte hierbei ausgeschlossen werden.

Insgesamt war auch in anderen Arbeiten die Bereitschaft der Patienten Fragen zur Visite vorzubringen gering. Beispielsweise ermittelte Raspe eine durchschnittliche Fragenanzahl von 1-1,5 pro Visite begründet mit tiefenpsychologisch und antropologisch zu erklärender Angst Fragen zur Visite zu formulieren [17]. Verglichen mit anderen Arbeiten konnten wir in dieser Studie eine noch niedrigere durchschnittliche Anzahl an Patientenfragen zur Visite ermitteln. Begründet sehen wir diese Tatsache, dass die CPs nicht nur ein probates Mittel darstellt die Arbeitsökonomie zur erhöhen sondern auch das Patienteninteresse zum Krankheitsbild der durchgeführten Operation und dem weiteren Krankheitsverlauf ausreichend zu decken.

Beispielsweise wurde das postoperative Interesse der Patienten zum Operationsinhalt über die CPs bereits präoperativ gesenkt, worauf nur noch etwas mehr als die Hälfte der Patienten ein weiteres Informationsbedürfnis auch postoperativ zeigte, wodurch insgesamt die Visitenzeiten gekürzt werden konnten.

Begründet sehen wir dies in der von Schuld et al. durchgeführten Arbeit, die den kausalen Zusammenhang zwischen strukturierten klinischen Abläufen, zu denen die chirurgische Aufklärung gehört und der Patientenzufriedenheit belegen [27]. Gerade in der Chirurgie stellt das persönliche Gespräch mit dem Patienten ein zentraler Bestandteil in dem Aufklärungsgespräch dar, in dem die wichtigsten Aspekte wie Rechtzeitigkeit, Inhalt sowie die Begleitumstände zu berücksichtigen sind [27]. Eine schriftliche multimedial gestützte Patientenaufklärung erhöht sowohl das Verständnis als auch die postoperative Zufriedenheit [27]. Die Auswertung der Arbeit ergab bei älteren Patienten eine höhere Zufriedenheit zwischen den zeitlichen Abläufen und 
der chirurgischen Aufklärung als jüngere Patienten [27]. Ebenfalls konnte bei längerem Patientenkontakt eine bessere Einschätzung der medizinischen Kompetenz des Arztes erfolgen mit ebenfalls erhöhter Patientenzufriedenheit [27].

\subsection{Umsetzung des Arbeitszeitgesetzes und Patientenzufriedenheit}

Vor Verabschiedung des neuen Arbeitszeitgesetzes zeigte die Arbeit von Rosta vom Herbst 2006 dass gerade in der Chirurgie die stärkste Arbeitsbelastung vorlag begründet durch außerplanmäßige Operationen, häufige Bereitschaftsdienste und nicht zuletzt durch den Ärztemangel [23].

Durch die strikten Auflagen des Arbeitszeitgesetzes mussten die Arbeitszeiten vor allem auf Ebene der Assistenzärzte gesetzeskonform gekürzt werden. Hinzu kam die erforderliche Einhaltung von Ruhezeiten nach den Bereitschaftsdiensten. All dies führte zu unregelmäßigen Besetzungen von Assistenzärzten zu den Routinevisiten. Die Besetzung der Visiten mit Oberärzten als konstante Entscheidungsträger und Informationsvermittler konnte zusammen mit der Eingliederung von CPs mit erhöhter Integration des Pflegepersonals die Visitenzeiten ohne negative Auswirkungen auf die Patientenzufriedenheit kürzen.

Durch konstante Besetzung der Visite mit Entscheidungsträgern konnte ein in der Arbeit von Hall beschriebenen Unterschied in der Patientenzufriedenheit abhängig vom Geschlecht und Ausbildungsgrad des Assistenzarztes vermieden werden. Beispielsweise belegte die Arbeit das gerinste Maß an Zufriedenheit von männlichen Patienten die von weiblichen Assistenzärztinnen zu Beginn ihrer Facharztausbildung betreut wurden [9].

Laut Lalouschek wurden kommunikative Fähigkeit der Ärzte bei der Interaktion mit den Patienten als sehr wichtig bewertet aber gleichzeitig als unspezifische Fertigkeiten vorausgesetzt, ohne sie in der ärztlichen Ausbildung zu berücksichtigen [11]. Es wird von den Ärzten ein partnerschaftlicher Dialog mit den Patienten gefordert, in dem der Patient nicht nur als „Kasus“ sondern als Kunde anzusehen ist [28]. Verschärft wird diese Tatsache noch durch Abschaffung des Arztes im Praktikum (AiP) nach erfolgtem Humanmedizinstudium und bestandenem Staatsexamen, und die damit verbundene sofortige Übernahme der klinischen Verantwortung im Rahmen der Visitenführung und des klinischen Arbeitsalltages [4]. 
Zahlreiche Arbeiten belegten deshalb Defizite in ärztlicher kommunikativer Fähigkeit. Werner et al. fordern daher explizit die Visitenführung bereits im Studium in gesonderten Unterrichtseinheiten den angehenden Ärzten zu vermitteln. Die Studenten sollen dabei die Verknüpfung von medizininhaltlichem Wissen mit Metakompetenzen wie Organisationsfähigkeit oder eigenverantwortliches Handeln in sogenannten Lernvisiten erlernen [34]. Tung erstellte in seiner Arbeit eine Hierarchie der Faktoren die zur Patientenzufriedenheit beitrugen. Bereits an 2. Stelle wurden die ärztlichen zwischenmenschlichen Fähigkeiten hinter den erstplazierten fachlichen Fähigkeiten eingestuft [33]. Die in den Interviews erhobene konstant hohe positive Zufriedenheit von über $80 \%$ während des gesamten Beobachtunszeitraum in beiden Gruppen sowie die meistattestierte positive Resonanz angenehme Gesprächsführung und Gesprächsatmosphäre auf die offene Frage „Was hat Ihnen heute bei Visite gut gefallen?" deckte sich mit den Erkenntnissen von Tung. Dies steht den Arbeiten entgegen, die kommunikative Defizite belegten [1, 2, 3, 9, 17, 30, 33].

\subsection{Fazit}

Entscheidend im Krankenhaus sind die Visiten, als gemeinsamer Begegnungspunkt in denen sich Patienten, Mediziner und gleichermaßen Pflegende direkt begegnen. Als Folge der Ökonomisierung im Gesundheitswesen und der strengeren Auflagen des Arbeitszeitgesetzes müssen sich Krankenhäuser und Kliniken heutzutage wesentlich gezielter mit effizienten Arbeitsmaßnahmen beschäftigen, als es noch vor zehn Jahren üblich war. Auch innerhalb der Universitätskliniken reicht es nicht mehr aus, sich weiterhin nur traditionell auf der Ebene hervorragender wissenschaftlicher Ergebnissen zu orientieren. Die erfolgreiche Integration von CPs kann die Kernbereiche Medizin, Pflege und Management langfristig und effizient vernetzen und somit die übergeordneten Zielsetzungen einer möglich hohen Patientenzufriedenheit mit medizinischer Spitzenqualität und einfach auswertbarer digitalisierter Datenbank vereinen. Sowohl bei kurzer wie auch langer Verweildauer der Patienten in unserer Klinik entstanden positive Bewertungen. Ausgehend davon, dass es gerade die Visiten sind, welche die häufigsten Kontakte zwischen Patienten 
und Krankenhauspersonal sind, hat es entscheidenden Anteil daran, wie der Patient letztlich seinen Krankenhausaufenthalt bewertet und weiter kommuniziert.

Trotz Umsetzung des neuen Arbeitszeitgesetzes vom Januar 2006 ist es unserer allgemeinchirurgischen Abteilung gelungen durch Etablierung von CPs und kompetente Visitenbesetzung Defizite in der Patientenzufriedenheit entgegenzuwirken. 


\section{Literatur}

1. Bartlett EE, Grayson M, Barker R, Levine DM, Golden A, Libber S (1984) The effect of physician communications skills on patient satisfaction; recall, and adherence. J Chron Dis. 37: 755-764

2. Blanchard CG, Ruckdeschel JC, Fletcher BA, Blanchard EB (1986) The Impact of Oncologists' Behavior on Patient Satisfaction With Morning Rounds. Cancer 58: 387-393

3. Bliesner Th, Köhle K (1986) Die ärztliche Visite - Chance zum Gespräch. Westdeutscher Verlag, Opladen

4. Dierkesmann R, Wladmann W (2010) Ärztliche Visite im Krankenhaus - Lernen aus der Vergangenheit? Klinikarzt 39: 332-336

5. Engelhardt K, Wirth A, Kindermann (1987) Kranke im Krankenhaus. Enke Verlag, Stuttgart

6. Fehlenberg D, Köhle K (1983) Die Stationsarztvisite zwischen Krankanhausroutine und therapeutischem Gespräch. Psychotherapie, Psychosomatik, Medizinische Psychologie 33: 45-52

7. Garcia-Gonzalez A, Gonzalez-Lopez L, Gamez-Nava JI, RodriguezArreola BE, Cox V, Suarez-Almazor ME (2009) Doctor-Patient Interactions in Mexican Patients With Rheumatic Disease. J Clin Rheumatol 15: 120-3

8. Geisler L (1978) Das Visitengespräch In: Arzt und Patient Begegnung im Gespräch. 3. Auflage. Pharma-Verlag, Frankfurt a. Main

9. Hall JA, Irisch JT, Roter DL, Ehrlich CM, Miller LH (1994) Satisfaction, Gender, and Communication in Medical Visits. Medical Care 32: 12161231 
10. Haferlach T (2008) Das Arzt-Patient Gespräch. Zuckschwerdt Verlag, München

11. Hellmich B, Palme R, Ade T, Leschke M (2010) Die klinische Visite und das ärztliche Gespräch - Sinnvolle Gestaltung unter aktuellen Krankenhausbedingungen. Klinikarzt 39: 344-348

12. Hollick J, Kerres A (2004) Pflegevisite - Ein Praxisleitfaden für Krankenpflege im OP - dienst und für die stationäre Kranken- und Altenpflege. Kohlhammer Verlag, Stuttgart

13. Lalouschek J (2002) Arzt - Patienten - Kommunikation In: Ärztliche Gesprächsausbildung eine diskursananlytische Studie zu Formen des ärztlichen Gespräches. Verlag für Gesprächsforschung, Radolfszell

14. Maaß E (1982) Anmerkungen zur Geschichte der klinischen Visite. In: Köhle K. Raspe H.H. Das Gespräch während der ärztlichen Visite. Urban Schwarzenberg, München-Wien-Baltimore

15. Middeke M (2008) Die Minutenzähler. Dtsch Med Wochenschr 133: 66

16. Nordmeyer J, Nordmeyer JP, Denke FW, Kerekjarto MV (1981) Formal-quantitative Aspekte des Sprachverhaltens von Arzt und Patient während der Visite. Med. Psychologie 11: 31-41

17. Nordmeyer J, Steinmann G, Denecke FW, Nordmeyer JP, Kerekjarto MV (1982) Verbale und nonverbale Kommunikation zwischen Problempatienten und Ärzten während der Visite. Med. Psychologie 8: 20-39

18. Papsdorf I, Hannick H, Trost F (2008) Information oder Verwirrung; Eine formal-quantitative Analyse augenärztlicher Visitengespräche. Ophthalmologe 15: 120-3 
19. Probst S (2007) Bedeutung der Visite in der Psychosomatischen Medizin, Dissertation Illertissen

20. Raspe HH (1980) Warum fragen Krankenhauspatienten so wenig? Eine medizinische Untersuchung der Stationsarztvisite. Therapiewoche 30: $560-573$

21. Raspe HH, Nordmeyer J (1981) Die Stationsvisite - eine verschenkte Möglichkeit? Therapiewoche 31: 1021-1025

22. Rassek M, Paar G, Schultheis KH, Simons C, Köhle K (1975) Funktion der ärztlichen Visite im Rahmen der internistisch-psychosomatischen Krankenversorgung. Verhandlungen der Deutschen Gesellschaft für Innere Medizin 81: 1735-1737

23. Rosta J (2007) Arbeitszeit der Krankenhausärzte in Deutschland. Dtsch Ärztebl 104: 2417-2424

24. Scherf W. Strach im psychotherapeutischen Prozess. In : Schneider H Sprache, Sprachentwicklung im psychotherapeutischen Prozess. 52. Psychotherapie-Seminar Freundenstadt, Mattes Verlag, Heidelberg

25. Schmidt C, Möller J, Reibe F, Günthert B, Kremer B (2003) Patientenzufriedenheit in der stationären Versorgung. Dtsch Med Wochenschr 128: 619-624

26. Schuld J, Richter S, Folz J, Jacob P, Gräber S, Schilling MK (2008) Influence of IT-supported clinical pathways on patient satisfaction at a surgical department of a university hospital. Dtsch Med Wochenschr 133: $1235-1239$ 
27. Schuld J, Moussavian MR, Frank B, Schmidt U.A., Kollmar O, Schilling MK (2010) Steigerung von Effizienz und Patientenzufriedenheit durch strukturierte Prozesse am Beispiel der Prämedikationsambulanz. Unfallchirurg 10: 1840-1846

28. Siebler M (2010) Die klinische und akademische Ausbildung im Jahre 2010 - Ärztemangel, Qualitätsverlust und „Deprofessionalisierung“? Klinikarzt 39: 356-359

29. Siegrist J (1978) Arbeit und Interaktion im Krankenhaus. Enke Verlag, Stuttgart

30. Simpson M, Buckmann R, Stewart M, Maguire P, Lipkin M, Novack D, Till J (1991) Doctor - patient communication: the Toronto consensus statement. BMJ 303: 1385-1387

31. Thiele W (2009) LASI Arbeitszeitgestaltung in Krankenhäusern Arbeitszeitproblematik am Beispiel des ärztlichen Dienstes LV 30 1-72

32. Töpfer A (2009) Marktorientierte Ausrichtung und Gestaltung aller Klinikaktivitäten In: Erfolgreiches Chancenmanagement im Krankenhaus: Das 15 Punkte Sofortprogramm für Kliniken. Springer Verlag, Heidelberg

33. Tung YC, Chang GM (2009) Patient satisfaction with recommendation of a primary care provider: associations of perceived quality and patient education. Int J Qual Health Care 21: 206-13

34. Werner A, Baur F, Zipfel S (2010) Ward rounds and didactic aspectsRealisation an d perspectives Klinikarzt 39: 350-355 
35.

Westphale C, Simons, Köhle K. (1983) Die Visite als psychotherapeutisches Gespräch. In: Heigl F, Neun H Psychotherapie in Klinik und Praxis, Bd. 2. Verlag für medizinische Psychologie, Vandenhoeck und Ruprecht, Göttingen und Zürich

36. Vogt M (2003) Visite als Steuerungselement in der Pflege und Therapie im Krankenhaus. Verlag Dr. Kovak, Hamburg 


\section{Abkürzungsverszeichnis}

$\begin{array}{ll}\text { AiP } & \text { Arzt im Pranktikum } \\ \text { CP } & \text { clinical pathway, Patientenpfad } \\ \text { ET } & \text { Entlassungstag } \\ \text { et al. } & \text { und andere } \\ \text { min } & \text { Minuten } \\ \text { MW } & \text { Mittelwert } \\ \text { OP } & \text { Operation } \\ \text { PE } & \text { Patientenerwartung } \\ \text { POT } & \text { postoperativer Tag } \\ \text { SEM } & \text { Standardfehler } \\ \text { u.a. } & \text { und andere }\end{array}$




\section{Abbildungsverzeichnis}

Abb. 1 Visitenlänge 29

Abb. 2 Patientenbegrüßung 31

Abb. 3 Patientenverabschiedung 33

Abb. 4 Körperliche Untersuchung 35

Abb. 5 Operationserklärung $\quad 37$

Abb. 6 Arzt-Patientgespräch 39

Abb. 7 Arztfragen pro Patient $\quad 41$

Abb. 8 Allgemeine Schmerzbefragung 43

Abb. 9 Patientenfragen pro Visite $\quad 45$

Abb. 10 Patientenzufriedenheit $\quad 47$ 


\section{Tabellenverzeichnis}

Tab. 1 Präoperative Patientenbefragung 16

$\begin{array}{ll}\text { Tab. } 2 \text { Visitenbeobachtung } & 18\end{array}$

$\begin{array}{ll}\text { Tab. } 3 \text { Patienteninterview } & 21\end{array}$

Tab. 4 Demographische Daten 26

$\begin{array}{ll}\text { Tab. } 5 \text { Klinische Angaben } & 27\end{array}$

$\begin{array}{ll}\text { Tab. } 6 \text { Geschlechtsvergleich } & 49\end{array}$ 


\section{Publikationen}

2009 Mitarbeit an einer Publikation Titel

"Arbeitszeitkonformes Visitenmodell und Patientenzufriedenheit“

Veigel S, Schmid A, Kollmar O, Schuld J, Bialas P, Kopp B, Schilling M.K., Moussavian M.R.

Zentralbl Chir 2010 accepted 


\section{Curriculum vitae}

— Persönliche Daten

Geburtsdaten

25.09.1978, Tübingen

Familienstand

ledig

Schulbildung

1985-1989

Grundschule Starzach

1989-1995

Realschule Horb a.N.

1995-1998

Wirtschaftsgymnasium Tübingen

Ausbildung

1998-2001

Berufsausbildung zur Chemielaborantin

Hygieneinstitut Universität Tübingen

2001-2008

Studium der Humanmedizin an der Universität des Saarlandes

- Praktisches Jahr

2007-2008 Praktisches Jahr an der Universitätsklinik des Saarlandes 
— Wissenschaftliche Tätigkeit

2009 klinische Doktorarbeit Titel

„Arbeitszeitkonformes Visitenmodell und Patientenzufriedenheit“ 


\section{Danksagung}

Diese Dissertationsarbeit stellte für mich eine besondere Herausforderung dar. Ich möchte mich bei allen Menschen bedanken, die zum Gelingen dieser Arbeit beigetragen haben.

Zunächst danke ich meiner Doktorvater Herrn Prof. Dr. Dr. med. Martin K. Schilling für die Überlassung des interessanten Themas dieser Arbeit sowie für die Möglichkeit den praktischen Teil dieser Arbeit auf einer seiner Stationen durchführen zu können.

Ganz besonders herzlich bedanken möchte ich mich bei meinem Betreuer Herrn OA Dr. Mohammed Reza Moussavian für die stetige Präsenz, Diskussionsbereitschaft und geduldigen Unterstützung in allen Phasen der Arbeit.

Bedanken möchte ich mich auch bei Herrn Peter Jacob für die Hilfe bei EDV-Fragen und bei Frau Berrit Kopp für die Hilfe in studienrechtlichen Belangen.

Außerdem möchte ich meinen Dank den Ärzten und dem Pflegepersonal der allgemeinchirurgischen Station CA-O1 des Universitätsklinikums des Saarlandes für die freundliche Zusammenarbeit während der Beobachtungsphase aussprechen.

Ein besonderer Dank gilt meinen Eltern, die mir mein Medizinstudium als auch die Anfertigung dieser Doktorarbeit ermöglicht haben. Sie haben mich immer unterstützt und haben immer an mich geglaubt. Deshalb widme ich diese Arbeit meinen stolzen Eltern. 